\title{
Nearly inviscid Faraday waves in annular containers of moderately large aspect ratio
}

\author{
José M. Vega , Edgar Knobloch , Carlos Martel \\ ETSI Aeronáuticos. Universidad Politécnica de Madrid, Plaza Cardenal Cisneros 3. 28040 Madrid. Spain \\ Department of Phrsics, University of Califonia. Berkeley, CA 94720. USA
}

\begin{abstract}
Nearly inviscid parametrically excited surface gravity-capillary waves in two-dimensional domains of finite depth and large aspect ratio are considered. Coupled equations describing the evolution of the amplitudes of resonant left- and right-traveling waves and their interaction with a mean flow in the bulk are derived, and the conditions for their validity established. Under suitable conditions the mean flow consists of an inviscid part together with a viscous mean flow driven by a tangential stress due to an oscillatory viscous boundary layer near the free surface and a tangential velocity due to a bottom boundary layer. These forcing mechanisms are important even in the limit of vanishing viscosity, and provide boundary conditions for the Navier-Stokes equation satisfied by the mean flow in the bulk. For moderately large aspect ratio domains the amplitude equations are nonlocal but decouple from the equations describing the interaction of the slow spatial phase and the viscous mean flow. Two cases are considered in detail, gravily-capillary waves and capillary waves in a microgravity environment.
\end{abstract}

PACS: $47.20 . \mathrm{Ky} ; 47.20 . \mathrm{Ma} ; 47.35 .+\mathrm{i} ; 47.54 .+\mathrm{r}$

Keywords: Faraday waves: Streaming flow: Gravity-capillary waves; Parametric resonance

\section{Introduction}

The Faraday system, i.e., the study of surface gravity-capillary waves excited parametrically by the vertical oscillation of a container, has attracted a great deal of attention [1-4]. Despite this a number of issues remain outstanding. This is largely due to the fact that existing theory fails to provide a quantitative description of the experimental results in containers of large aspect ratio. One possible explanation, pursued by us in several papers $[5,6]$ focuses on the fact that these theories include only the leading order effects of viscosity $[7,8]$ despite the fact that for typical experimental parameter values this approach predicts an incorrect viscous dissipation time in the absence of parametric forcing. This is because the dissipation time for Faraday waves excited by typical oscillation frequencies is in fact dominated by dissipation in the bulk of the domain, and not in the boundary layers at solid walls 
as usually assumed. However, there is an additional important effect associated with the presence of viscosity that illustrates the singular nature of the required perturbation theory. This effect arises because the oscillatory viscous boundary layers at the free surface and the bottom of the container (as well as any lateral boundaries, if present) are capable of driving a large scale mean flow, hereafter a viscous mean flow or a streaming flow, due to a nonzero (time-averaged) Reynolds stress in these boundary layers. These flows have either been entirely ignored in the past or treated in an incomplete or inconsistent manner, but they are important because they can interact nontrivially with the surface waves responsible for them. This is so, for example, in systems of small to moderate aspect ratio provided at least two modes of oscillation are excited [9,10]. Large aspect ratio systems are yet more subtle because of the presence of an additional, inviscid mean flow. For inviscid free waves this mean flow is associated with spatial modulation of a single mode, as described by the celebrated Davey-Stewartson equations $[11,12]$. If viscosity is retained and the system forced, as in a shear flow, a similar set of equations but with complex coefficients can be derived [13]. In general the mean flow present will contain both types of contributions, even in nearly inviscid flows.

This paper is devoted to the derivation of the following equations governing the interaction between two parametrically excited counterpropagating wavetrains and the associated mean flow in a two-dimensional, annular Faraday system,

$$
\begin{aligned}
A_{t}-v_{\mathrm{g}} A_{x}= & \mathrm{i} \alpha A_{x x}-(\delta+\mathrm{i} d) A+\mathrm{i}\left(\alpha_{3}|A|^{2}-\alpha_{4}|B|^{2}\right) A+\mathrm{i} \alpha_{5} \mu \bar{B}+\mathrm{i} \alpha_{6} \int_{-1}^{0} g(y)\left\langle\psi_{y}^{\mathrm{m}}\right\rangle^{x} \mathrm{~d} y A \\
& +\mathrm{i} \alpha_{7}\left(f^{\mathrm{m}}\right\rangle^{x} A+\mathrm{IIOT}, \\
B_{l}+v_{\mathrm{g}} B_{x}= & \mathrm{i} \alpha B_{x x}-(\delta+\mathrm{i} d) B+\mathrm{i}\left(\alpha_{3}|B|^{2}-\alpha_{4}|A|^{2}\right) B+\mathrm{i} \alpha_{5} \mu \bar{A}-\mathrm{i} \alpha_{6} \int_{-1}^{0} g(y)\left\langle\psi_{y}^{\mathrm{m}}\right\rangle^{x} \mathrm{~d} y B \\
& +\mathrm{i} \alpha_{7}\left(f^{\mathrm{m}}\right)^{x} B+\mathrm{HOT}, \\
A(x+L, \ell) \equiv & A(x, l), \quad B(x+L, \ell) \equiv B(x, l),
\end{aligned}
$$

together with the conditions under which these equations provide the correct description of Faraday waves in systems with reflection symmetry and one extended dimension. IIere $L \gg 1$ is the aspect ratio of the system, measured in units of the layer depth. As part of the derivation explicit expressions for the coefficients are obtained. The complex amplitudes $A$ and $B$ are the amplitudes of the two counterpropagating waves driven parametrically by the forcing (with dimensionless amplitude $\mu$ ), and the notation IIOT indicates higher order terms. The first seven terms in these equations, accounting for inertia, propagation at the group velocity $v_{\mathrm{g}}$, dispersion, damping, detuning, cubic nonlinearity and parametric forcing, are familiar from existing weakly nonlinear, nearly inviscid theories [14]. The last two terms account for coupling to the mean flow in the bulk (indicated by the superscript $\mathrm{m}$ ) and are conservative. They are written in terms of (a local average (\rangle$^{x}$ of ) the streamfunction $\psi^{\mathrm{m}}$ for the mean flow and the associated free surface elevation $f^{\mathrm{m}}$. These quantities evolve according to the equations

$$
\begin{aligned}
& \psi_{x x}^{\mathrm{m}}+\psi_{y y}^{\mathrm{m}}=\Omega^{\mathrm{m}}, \quad \Omega_{t}^{\mathrm{m}}-\left\lceil\psi_{y}^{\mathrm{m}}+\left(|A|^{2}-|B|^{2}\right) g(y)\right\rceil \Omega_{x}^{\mathrm{m}}+\psi_{x}^{\mathrm{m}} \Omega_{y}^{\mathrm{m}}=C_{g}\left(\Omega_{x x}^{\mathrm{m}}+\Omega_{y y}^{\mathrm{m}}\right)+\text { HOT, } \\
& \psi_{x}^{\mathrm{m}}-f_{t}^{\mathrm{m}}=\beta_{1}\left(|B|^{2}-|A|^{2}\right)_{x}+\text { HOT }, \quad \psi_{y y}^{\mathrm{m}}=\beta_{2}\left(|A|^{2}-|B|^{2}\right)+\text { HOT } \quad \text { al. } y=0, \\
& (1-S) f_{x}^{\mathrm{m}}-S f_{x x x}^{\mathrm{m}}-\psi_{y i}^{\mathrm{m}}+C_{g}\left(\psi_{y y y}^{\mathrm{m}}+3 \psi_{x x y}^{\mathrm{m}}\right)=-\beta_{3}\left(|A|^{2}+|B|^{2}\right)_{x}+\text { HOT } \quad \text { al. } y=0, \\
& \int_{0}^{L} \Omega_{y}^{\mathrm{m}} \mathrm{d} x=\psi^{\mathrm{m}}=0, \quad \psi_{y}^{\mathrm{m}}=-\beta_{4}\left\lceil\mathrm{i} A \bar{B} \mathrm{c}^{2 i k x}+\text { c.c. }+|B|^{2}-|A|^{2}\right\rceil+\text { HOT } \quad \text { al. } y=-1,
\end{aligned}
$$




$$
\begin{aligned}
& \psi^{\mathrm{m}}(x+L, y, l) \equiv \psi^{\mathrm{m}}(x, y, l), \quad f^{\mathrm{m}}(x+L, t) \equiv f^{\mathrm{m}}(x, l), \\
& \int_{0}^{L} f^{\mathrm{m}}(x, t) \mathrm{d} x=0,
\end{aligned}
$$

valid oulside of viscous boundary layers at the free surface and the bottom $(y=-1)$. Here $C_{g} \ll 1$ is a dimensionless measure of viscosily. The resulting equalions differ from the exact equalions forming the starling point for the analysis in the presence of the forcing terms in the boundary condilions (1.5)-(1.7) and in two essenlial simplificalions: the fast oscillation associated with the surface waves has been filtered oul, and the boundary conditions are applied at the unperlurbed location of the free surface, $y=0$. The mean flow itself is forced in lwo ways. The right-hand sides of the boundary conditions (1.5a) and (1.6) provide a normal forcing mechanism; this mechanism is the only onc present in the striclly inviscid case and does not appear unless the aspect ratio is large. The right-hand sides of the boundary conditions (1.5b) and (1.7c) describe two shear forcing mechanisms, a tangenlial stress at the free surface and a langenlial velocily at the bottom wall. Note that neither of these forcing terms vanishes in the limil of small viscosily (i.c., as $C_{g} \rightarrow 0$ ), ci. [15,16], in contrast to the strictly intiscid theory in which terms of this lype do not arise.

The gencral coupled amplitude-mean-flow (hereafter (FCAMF) equalions summarized above are derived here by means of a consistent expansion that treats both the viscosily (i.c., the parameler $C_{g}$ ) and the inverse aspecl ralio $L^{-1}$ of the system as independenl small parameters. However, in particular and physically relevant regimes in which these parameters are linked, the GCAMF equations simplify further. $\Lambda$ parlicularly useful sim-

plification arises when the system is large but not too large, in the sense that $L \ll C_{g}^{-1 / 2}$. In this regime, two cases are of special interest, corresponding, respectively, to nearly inviscid gravity-capillary waves and to pure capillary waves in a microgravity environment. Both systems are described by nonlocal amplitude equations of the type already studied in [17]; these equations determine the surface waves up to a spatial phase and decouple from the remaining equations governing the interaction between this phase and the (viscous) mean flow. If the system size is too large, different (hyperbolic) equations apply, but these are not discussed here (see $[18,19]$ for a related problem). The remainder of this paper is organized as follows. In Section 2, we formulate the basic equations and explain the nature of the analysis that leads to the GCAMl equations. This analysis is performed explicitly in Section 3, with the simplifications alluded to above carried out in Section 4. The detailed properties of the resulting equations can only be ascertained numerically, and will be described in subsequent work. The paper concludes with a brief discussion in Section 5. Certain details of the analysis of the oscillatory boundary layers at the top and bottom of the layer that are required in the body of the paper can be found in Appendix A.

\section{Formulation and other preliminaries}

As a model of Faraday waves itı antular containers, we consider a lwo-dimensional, laterally unbounded fluid layer above a horizontal plate that is vibrated verlically with an approprialely small amplitude. We use a Cartesian coordinate system with the $x$-axis along the unperturbed free surface and $y$ verlically upwards, and nondimensionali\%e space and lime with the unperturbed depth $h$ and the gravily-capillary lime $\left[g / h+T /\left(\rho h^{3}\right)\right]^{-1 / 2}$, where $g$ is the gravitational acceleration, $\rho$ the density and $T$ the coefficient of surface Iension. The nondimensional equations governitig the system then are

$$
\begin{aligned}
& \psi_{x x}+\psi_{y y}=\Omega, \quad \Omega_{t}-\psi_{y} \Omega_{x}+\psi_{x} \Omega_{y}=C_{g}\left(\Omega_{x x}+\Omega_{y y}\right) \\
& f_{t}-\psi_{x}-\psi_{y} f_{x}=\left(\psi_{y y}-\psi_{x x}\right)\left(1-f_{x}^{2}\right)-4 f_{x} \psi_{x y}=0 \quad \text { at. } y=f
\end{aligned}
$$




$$
\begin{aligned}
& (1-S) f_{x}-S\left(\frac{f_{x}}{\sqrt{1+f_{x}^{2}}}\right)_{x x}-\psi_{y t}+\psi_{x t} f_{x}-\left(\psi_{x}+\psi_{y} f_{x}\right) \Omega+\frac{1}{2}\left(\psi_{x}^{2}+\psi_{y}^{2}\right)_{x}+\frac{1}{2}\left(\psi_{x}^{2}+\psi_{y}^{2}\right)_{y} f_{x} \\
& -4 \mu \omega^{2} \cos (2 \omega l) f_{x}=-C_{g}\left[3 \psi_{x x y}+\psi_{y y y}-\left(\psi_{x x x}+\psi_{x y y}\right) f_{x}\right\rfloor+2 C_{g}\left[\frac{2 \psi_{x y} f_{x}^{2}+\left(\psi_{x x}-\psi_{y y}\right) f_{x}}{1+f_{x}^{2}}\right]_{x} \\
& +2 C_{g} \frac{\left(\psi_{x x y}-\psi_{y y y}\right) f_{x}^{2}-\psi_{x y y}\left(1-f_{x}^{2}\right) f_{x}}{1+f_{x}^{2}} \text { at } y=f, \\
& \int_{0}^{L} \Omega_{y} \mathrm{~d} x=\psi=\psi_{y}=0 \quad \text { at } y=-1, \\
& \int_{0}^{L} \int \mathrm{d} x=0,
\end{aligned}
$$

subject to the requirement that $\psi$ and $f$ are both periodic in $x$ with spatial period $L$ (the nondimensional length of the annulus). IIere $\psi$ is the streamfunction, such that the velocity $(u, v)=\left(-\psi_{y}, \psi_{x}\right), \Omega$ the vorticity, and $f$ the free surface deflection required to satisfy volume conservation recalled in (2.5). The boundary condition (2.4a) is necessary in order that the pressure be periodic in $x$. The resulting problem depends on the aspect ratio $L$, the nondimensional vibration amplitude $\mu$ and frequency $2 \omega$, the capillary-gravity number $C_{g}=v /\left[g h^{3}+(T h / \rho)\right]^{1 / 2}$ and the gravity-capillary balance parameter $S=T /\left(T+\rho g h^{2}\right)$, where $v$ is the kinematic viscosity. Note that $C_{g}$ and $S$ are related to the usual capillary number $C=v[\rho / T h]^{1 / 2}$ and the Bond number $B=\rho g h^{2} / T$ by

$$
c_{g}=\frac{C}{(1+B)^{1 / 2}}, \quad S=\frac{1}{1+B} .
$$

Thus

$$
0 \leq S \leq 1
$$

and the extreme values $S=0,1$ correspond to the purely gravitational $(T=0)$ and the purely capillary $(g=0)$ limits, respectively.

The basic assumption made below is that viscosity is small, namely

$$
C_{g} \ll 1
$$

To understand the origin of the nearly inviscid and viscous mean flows in this limil., il suffices to look al the spectrum of the unforced problem, linearized around $\psi=f=0$ [5]. The normal modes take the form

$$
(\psi, f)=(\psi, F) \mathrm{c}^{\lambda t+\mathrm{i} k x}
$$

In general, when $C_{g} \ll 1$ there are two types of such modes:

(A) The nearly inviscid modes (or surface modes) obey the dispersion relation

$$
\lambda=\mathrm{i} \omega-(1+\mathrm{i}) \alpha_{1} C_{g}^{1 / 2}-\alpha_{2} C_{g}+O\left(C_{g}^{3 / 2}\right),
$$

where

$$
\omega=\left[\left(1-S+S k^{2}\right) k \tanh k\right]^{1 / 2}, \quad \alpha_{1}=\frac{k(\omega / 2)^{1 / 2}}{\sinh (2 k)}, \quad \alpha_{2}=\left[2+\frac{5+3 \tanh ^{2} k}{16 \sinh ^{2} k}\right] k^{2} .
$$

Lq. (2.10) provides a good approximation for both the frequency $\operatorname{Im}(\lambda)$ and the damping rate

$$
\delta \equiv-\operatorname{Re}(\hat{\lambda})=\alpha_{1} C_{g}^{1 / 2}+\alpha_{2} C_{g}
$$



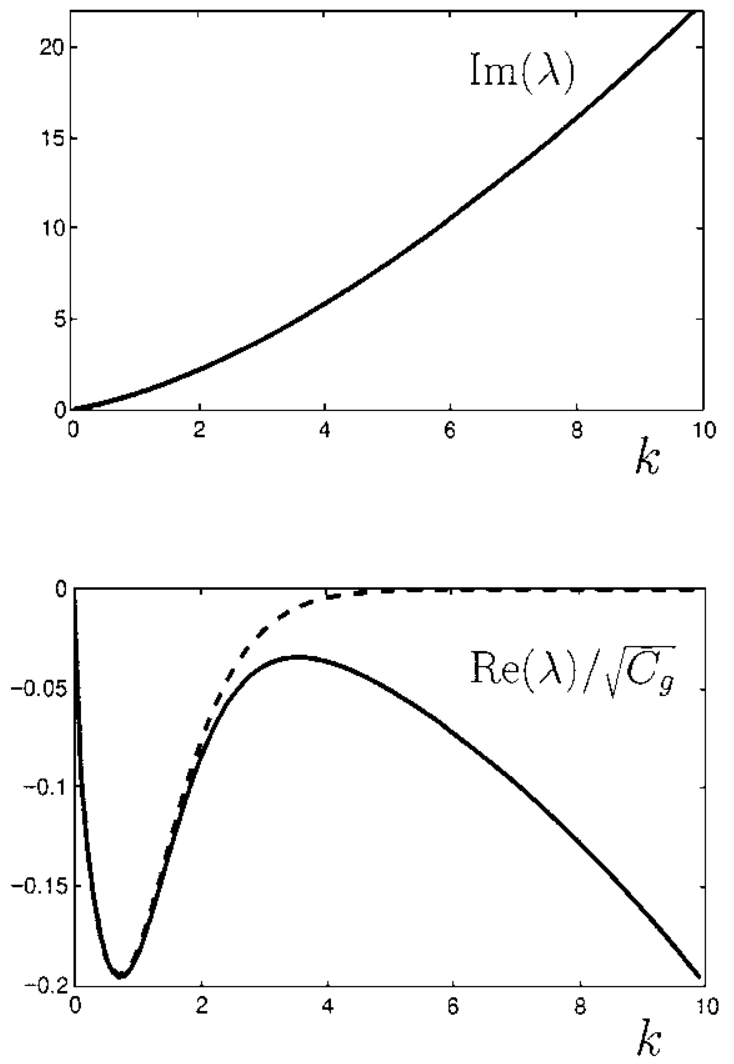

Fig. I. The nearly inviscid dispersion relation, $\operatorname{Im} \lambda$ and $\operatorname{Re} \lambda$ vs. $k$ for $C_{k^{\prime}}=10^{-6}, S=0.5$, from kq. $(2.10)$ using the $O\left(C_{g}^{1 / 2}\right)$ results (dashed line) and the $O\left(C_{g}\right)$ results (solid line).

for small but fixed values of $C_{\beta}$, see Fig. 1. However, as noted in [5], if the third term in (2.10) is omitted the resulting approximation breaks down as soon as $k \gtrsim k_{\mathrm{m}} \sim\left|\ln C_{\xi}\right|$. Since these moderately large values of $k$ are also of interest this term will be retained in what follows.

The eigenfunction associated with the dispersion relation (2.10) is given (up to a constant factor) by

$$
(\Psi, F)=\left(\Psi_{0}, 1\right)+O\left(C_{g}^{1 / 2}\right) \quad \text { with } \Psi_{0}=\frac{\omega \sinh [k(y+1)]}{k \sinh k} .
$$

These modes therefore exhibit a significant free-surface deflection and are irrotational in the bulk, outside two thin boundary layers (whose thickness is $\left.O\left(C_{g} / \omega\right)^{1 / 2}\right)$ attached to the bottom plate and the free surface. For small $C_{g}$ their decay rate is $O\left(C_{g}^{1 / 2}\right)$, i.e., these modes are all near-marginal. Note that the horizontal wavenumber $k$ is only restricted by the periodicity condition and thus can take any value of the form $2 \pi N / L$, where $N$ is an integer; in the limit $L \rightarrow \infty$ the allowed wavenumbers become dense on the real line. However, the assumption (2.16) implies that the relevant nearly inviscid modes are either of long wavelength or are concentrated around the two counterpropagating modes. The long wave modes constitute the nearly inviscid mean flow; in the strictly inviscid case, this flow is the mean flow considered in inviscid theories [11,12]. However, because of its long wavelength this mean flow does not appear if the aspect ratio is of order unity $[9,10]$.

(B) The viscous modes (or hydrodynamical modes) obey the dispersion relation

$$
\lambda=-C_{\xi}\left[k^{2}+q_{n}(k)^{2}\right]+\mathrm{O}\left(C_{\xi}^{2}\right),
$$


where for each $k>0, q_{n}>0$ is the $n$th root of $q \tanh k=k \tan q$, and hence decay on an $O\left(C_{g}\right)$ timescale, i.e., more slowly than the surface modes when $C_{g}$ is sufficiently small. Consequently, these modes are also near-marginal. Since the associated eigenfunction is

$$
\Psi=\sin q_{n} \sinh (k y)-\sinh k \sin \left(q_{n} y\right)+\mathrm{O}\left(C_{g}\right), \quad F=\mathrm{O}\left(C_{g}\right)
$$

these modes do not result in any significant. free-surface deformalion at leading order. On the other hand, they are rotational throughout the domain and when foreed at the edge of the oscillatory boundary layers atlached to the bothom plate and the liee surface by the mechantisms deseribed by Schlichting [20] and Ionguel-Higgins [21] (see $\Lambda$ ppendix $\Lambda$ ), they constitule the viscous mean flow. In view of its slow decay this flow must be included in anly realistic nearly inviscid description. The assumption that follows implies that the relevant viscous modes are concenlrated around a discrete set of values of $k$.

\subsection{Basic assumption}

The spatial Fourier tratssforms of the oscillatory part (in lime) of $\psi$ and $f$ peak for all lime around the wavenumbers $\pm k$, while those of the nonoscillatory part peak at wavenumbers $\pm 2 m k$, with $m=0,1, \ldots$.

Here and hereafter $k$ denotes the wavenumber of the parametrically exciled surface mode. If $L$ is not too large, as specified in Eq. (2.19), this assumption is consistent, in the sense that the resulting equations do not generate arbitrarily small scales. This properly is not guaranteed for larger $L$.

In addition to this assumplion we also assume that

$$
\left|\psi_{x}\right|+\left|\psi_{y}\right| \ll 1, \quad|f| \ll 1, \quad C_{g} \ll 1, \quad L^{-1} \ll 1,
$$

i.e., we focus on weakly nonlinear nearly inviscid waves in large aspect ratio systems. This restriction requires, in addition, that $\mu \ll 1$. Moreover, in view of the comment after Lq. (2.12), we also assume that

$$
1 \lesssim k \lesssim\left|\ln C_{g}\right|
$$

which implies that $\delta=O\left(C_{g}\right)^{1 / 2} \ll 1(\sec (2.12))$ and $1 \lesssim \omega \lesssim\left\lceil(1-S)\left|\ln C_{g}\right|+S\left|\ln C_{g}\right|^{3}\right]^{1 / 2}(\sec (2.11))$. As explained in Seclion 5, this assumplion can be relaxed.

Within these assumplions several essentially different distinguished limits are possible, depending on the relative values of the small paramelers $C_{g}, \mu$ and $L^{-1}$, and also on the order of magnilude of $1-S$. In this paper, we assume that $L$ is not too large, in the sense that.

$$
1 \ll L \ll \frac{v_{\mathrm{g}}}{\delta+|d|+\mu},
$$

where $v_{\mathrm{g}}, \delta$ and $d$ are the (nondimensional) group velocity, damping rate and detuning of the surface waves, defined by (3.24), (2.12) and (3.28), respectively, and consider separately the two cases $S \ll 1$ and $S \sim 1$ (see Section 4 ).

\section{The general coupled amplitude-mean flow equations}

In the derivation that follows il is convenienl to treal the small parameters $C_{g}$ and $L^{-1}$ as unrelated. Since viscosily is small, we must distinguish three regions in the physical domain, namely, the two oscillatory boundary layers 


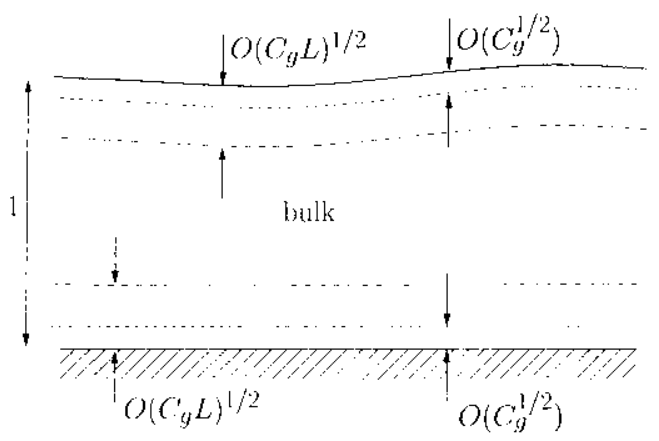

Fig. 2. Sketch of the primary and secondary boundary layers, indicating their widths in comparison to the layer depth.

(of thickness $O\left(C_{g}^{1 / 2}\right)$ ) mentioned in Section 2 and the remaining part (or bulk) of the domain (see Fig. 2). The boundary layers must be considered in order to obtain the correct boundary conditions for the solution in the bulk. The description of these boundary layers can be found in Appendix A.

Now, according to assumption (2.16), the streamfunction in the bulk and the free surface deflection can be decomposed into three parts, namely: (i) the two counterpropagating wavetrains mentioned in Section 2, which are slowly modulated both in space and time around a basic frequency $\omega$ and wavenumbers $\pm k$; (ii) a mean flow, which depends weakly on time but can exhibit significant dependence on the space variables $x$ and $y$; and (iii) the remaining part of the solution, which will be called nonresonant. Since we are not distinguishing between inviscid and viscous mean flows we must allow the mean flow to exhibit a significant dependence on the horizontal coordinate $x$. This is because the mean flow must include, among other things, any viscous modes with $O(k)$ wavenumber associated with the basic wavetrain. The assumption (2.16) is equivalent to the requirement that the mean flow variables exhibit well-defined averages in the fast variable $x$ (see (3.19)).

Under these conditions the free-surface deflection, and the streamfunction and vorticity in the bulk, can be written in the form

$$
\begin{aligned}
f= & \mathrm{e}^{\mathrm{i} \omega t}\left(A \mathrm{e}^{\mathrm{i} k x}+B \mathrm{e}^{-\mathrm{i} k x}\right)+\gamma_{1} A \bar{B} \mathrm{e}^{2 \mathrm{i} k x}+\gamma_{2} \mathrm{e}^{2 \mathrm{i} \omega t}\left(A^{2} \mathrm{e}^{2 \mathrm{i} k x}+B^{2} \mathrm{e}^{-2 \mathrm{i} k x}\right)+f^{+} \mathrm{e}^{\mathrm{i} \omega t+\mathrm{i} k x}+f^{-} \mathrm{e}^{\mathrm{i} \omega t-\mathrm{i} k x} \\
& + \text { c.c. }+f^{\mathrm{m}}+\mathrm{NRT}, \\
\psi= & \Psi_{0} \mathrm{e}^{\mathrm{i} \omega t}\left(A \mathrm{e}^{\mathrm{i} k x}-B \mathrm{e}^{-\mathrm{i} k x}\right)+\gamma_{3} \Psi_{22} \mathrm{e}^{2 \mathrm{i} \omega t}\left(A^{2} \mathrm{e}^{2 \mathrm{i} k x}-B^{2} \mathrm{e}^{-2 \mathrm{i} k x}\right)+\psi^{+} \mathrm{e}^{\mathrm{i} \omega t+\mathrm{i} k x}+\psi^{-} \mathrm{e}^{\mathrm{i} \omega t-\mathrm{i} k x} \\
& + \text { c.c. }+\psi^{\mathrm{m}}+\mathrm{NRT}, \\
\Omega= & \mathrm{i} \omega^{-1} \mathrm{e}^{\mathrm{i} \omega t}\left[\left(A \mathrm{e}^{\mathrm{i} k x}-B \mathrm{e}^{-\mathrm{i} k x}\right) \Psi_{0}^{\prime} \Omega_{x}^{\mathrm{m}}-\mathrm{i} k\left(A \mathrm{e}^{\mathrm{i} k x}+B \mathrm{e}^{-\mathrm{i} k x}\right) \Psi_{0} \Omega_{y}^{\mathrm{m}}+\text { HOT }\right]+\text { c.c. }+\Omega^{\mathrm{m}}+\mathrm{NRT} .
\end{aligned}
$$

Here the superseript $m$ denotes terms associated with the mean flow, NRT denotes nonresonant terms and HOT denotes higher order terms. The function $\Psi_{0}$ is defined in (2.13). Moreover, the quantities $A, B, f^{ \pm}$and $\psi^{ \pm}$must all depend weakly on $t$ and $x$, while $f^{\mathrm{m}}, \psi^{\mathrm{m}}$ and $\Omega^{\mathrm{m}}$ depend weakly on $t$ but strongly on $x$ (ef. Eq. (1.7)), i.e.,

$$
\begin{aligned}
& \left|A_{x}\right|+\left|A_{t}\right| \ll|A| \ll 1, \quad\left|B_{x}\right|+\left|B_{t}\right| \ll|B| \ll 1, \\
& \left|f_{x}^{ \pm}\right|+\left|f_{t}^{ \pm}\right| \ll\left|f^{ \pm}\right| \ll 1, \quad\left|\psi_{x}^{ \pm}\right|+\left|\psi_{t}^{ \pm}\right| \ll\left|\psi^{ \pm}\right| \ll 1, \\
& \left|f_{t}^{\mathrm{m}}\right| \ll\left|f^{\mathrm{m}}\right| \ll 1, \quad\left|\psi_{t}^{\mathrm{m}}\right| \ll\left|\psi^{\mathrm{m}}\right| \ll 1, \quad\left|\Omega_{t}^{\mathrm{m}}\right| \ll\left|\Omega^{\mathrm{m}}\right| \ll 1,
\end{aligned}
$$

while the periodic boundary equations on $\psi$ and $f$ imply that

$$
A(x+L, t) \equiv \mathrm{e}^{-2 \mathrm{i} k L} A(x, t), \quad B(x+L, t) \equiv \mathrm{e}^{2 \mathrm{i} k L} B(x, t) .
$$


The terms proportional to $\mathrm{e}^{\mathrm{i} \omega t \pm \mathrm{i} k x}$ describe the two counterpropagating wavetrains. In order to distinguish between the leading order and higher order contributions to these waves we impose the additional condition

$$
\int_{-1}^{0} \psi^{ \pm}(x, y, t) \mathrm{d} y=0
$$

for all $x$ and $t$. This condition serves as a definition of the complex amplitudes $A$ and $B$, and readily implies that

$$
\left|f^{+}\right|+\left|\psi^{+}\right| \ll|A|, \quad\left|f^{-}\right|+\left|\psi^{-}\right| \ll|B| .
$$

The coefficienls $\gamma_{1}, \gamma_{2}, \gamma_{3}$ and the funclion $\Psi_{22}$ in (3.1) and (3.2) are given by

$$
\begin{aligned}
& \gamma_{1}=\frac{\left(\sigma^{2}+1\right) \omega^{2}}{\sigma^{2}\left(1-S+4 S k^{2}\right)}, \\
& \gamma_{2}=\frac{\left(3-\sigma^{2}\right) k\left(1-S+S k^{2}\right)}{2 \sigma\left[(1-S) \sigma^{2}-S k^{2}\left(3-\sigma^{2}\right)\right]}, \\
& \gamma_{3}=\frac{3 \omega\left[(1-S)\left(1-\sigma^{2}\right)+S k^{2}\left(3-\sigma^{2}\right)\right]}{2 \sigma\left[(1-S) \sigma^{2}-S k^{2}\left(3-\sigma^{2}\right)\right]}, \\
& \Psi_{22}=\frac{\sinh [2 k(y+1)]}{\sinh (2 k)}
\end{aligned}
$$

where $\sigma=\operatorname{lath} k$. Note that $\gamma_{2}$ and $\gamma_{3}$ diverge al $(1-S) \sigma^{2}=S k^{2}\left(3-\sigma^{2}\right)$, i.c., when the strictly inviscid eigenlfrequency (2.11a) salisfies $\omega(2 k)=2 \omega(k)$. In the present paper, we do not pursue this resonante further; sec $[22,23]$ for a strictly inviscid analysis, and $[24-26]$ for nearly inviscid descriptions that ignore mean flow.

Substiluling (3.1)-(3.7) into (2.1)-(2.5) with the boundary conditions derived in $\Lambda$ ppendix $\Lambda$, we oblain the evolution equalions and boundary conditions for $\psi^{\mathrm{m}}, \Omega^{\mathrm{m}}, f^{\mathrm{m}}$ listed in (1.4)-(1.9), together wilh the followitg equations and boundary condilions for the perlurbations $\psi^{+}, f^{+}$:

$$
\begin{aligned}
& \psi_{y y}^{+}-k^{2} \psi^{+}=-2 \mathrm{i} k\left(\Psi_{0} \Lambda_{x}+\psi_{x}^{+}\right)-\Psi_{0} \Lambda_{x x}-\left(\frac{k}{\omega}\right) \Psi_{0}\left\langle\Omega_{y}^{\mathrm{m}}\right\rangle^{x} \Lambda+\text { IIOT } \quad \text { in }-1<y<0, \\
& \mathrm{i} \omega f^{+}-\mathrm{i} k \psi^{+}=-\Lambda_{t}+\Psi_{0} \Lambda_{x}+\psi_{x}^{+}+\left[\mathrm{i} k\left(\beta_{5}|A|^{2}+\beta_{6}|B|^{2}\right)+\mathrm{i} k \Psi_{0}^{\prime}\left\langle f^{\mathrm{m}}\right\rangle^{x}\right. \\
& \left.+\mathrm{i} k\left\langle\psi_{y}^{\mathrm{m}}\right\rangle^{x}+\beta_{y} C_{g}\right\rfloor A+\mathrm{HOI} \text { at } y=0 \text {, } \\
& \mathrm{i} k\left(1-S+S k^{2}\right) f^{+}-\mathrm{i} \omega \psi_{y}^{+}=3 \mathrm{i} k S A_{x x}+\Psi_{0}^{\prime} A_{t}+\mathrm{i} k\left(\beta_{7}|A|^{2}+\beta_{S}|B|^{2}\right) A+C_{g}\left(3 k^{2} \Psi_{0}^{\prime}-\Psi_{0}^{\prime \prime \prime}\right) A-\mathrm{i} k \mu \omega^{2} \bar{B} \\
& +\left[\mathrm{i} \omega \Psi_{0}^{\prime \prime}\left(f^{\mathrm{m}}\right)^{x}+\mathrm{i} k \Psi_{0}\left\langle\Omega^{\mathrm{m}}\right\rangle^{x}-\mathrm{i} k \Psi_{0}^{\prime}\left\langle\psi_{y}^{\mathrm{m}}\right)^{x}\right] \Lambda+\mathrm{IIOT} \text { at } y=0 \text {, } \\
& \psi^{+}=\left\lfloor(1+\mathrm{i}) \beta_{10} C_{g}^{1 / 2}+\beta_{11} C_{g}\right\rfloor A+\mathrm{HOI}^{\prime} \text { at } y=-1 \text {, } \\
& \psi^{+}{ }^{+}(x+L, y, t) \equiv \psi^{+}(x, y, t), \quad f^{+}(x+L, t) \equiv f^{+}(x, t) .
\end{aligned}
$$

IIere the mean value ()$^{x}$ is defined by

$$
\langle G(x, y, t)\}^{x}=(2 \ell)^{-1} \int_{x-\ell^{\prime}}^{x+\ell} G(z, y, t) \mathrm{d} z \quad \text { with } 1 \ll \ell \ll L,
$$


and is required to be independent of $\ell$. In view of the assumption (2.16) this average is well-defined and can be thought of as a filter that filters out the smallest scales, $x \sim 1$. lor this reason $\langle G(x, y, l)\rangle^{x}$ may still depend on the horizontal coordinate $x$, albeit weakly, so that

$$
\left\langle f_{x}^{\mathrm{m}}\right\rangle^{x} \ll\left\langle f^{\mathrm{ml}}\right\rangle^{x}, \quad\left\langle\psi_{x}^{\mathrm{m}}\right\rangle^{x} \ll\left\langle\psi_{y}^{\mathrm{m}}\right\rangle^{x}, \quad\left\langle\Omega_{x}^{\mathrm{m}}\right\rangle^{x} \ll\left\langle\Omega_{y}^{\mathrm{m}}\right\rangle^{x} .
$$

These estimates have been used to drop higher order terms.

The coefficients $\beta_{1}, \ldots, \beta_{S}$ and the function $g$ in $(1.4)-(1.7),(3.15)$ and (3.16) are given by

$$
\begin{aligned}
& \beta_{1}=\frac{2 \omega}{\sigma}, \quad \beta_{2}=\frac{8 \omega k^{2}}{\sigma}, \quad \beta_{3}=\frac{\left(1-\sigma^{2}\right) \omega^{2}}{\sigma^{2}}, \quad \beta_{4}=\frac{3\left(1-\sigma^{2}\right) \omega k}{\sigma^{2}} \\
& \beta_{5}=\frac{\gamma_{2} \omega}{\sigma}+\frac{\gamma_{3} k\left(1+\sigma^{2}\right)}{\sigma}+\frac{3 \omega k}{2}, \quad \beta_{6}=-\frac{\gamma_{1} \omega}{\sigma}-\omega k, \quad \beta_{7}=\gamma_{2} \omega^{2}+\frac{\gamma_{3} \omega k\left(\sigma^{2}-1\right)}{\sigma^{2}}-\frac{5 \omega^{2} k}{2 \sigma}+\frac{3 S k^{4}}{2} \\
& \beta_{5}=\gamma_{1} \omega^{2}+\frac{3 \omega^{2} k}{\sigma}+3 S k^{4}, \quad g(y)=\frac{2 \omega k \cosh \lceil 2 k(y+1)\rceil}{\sinh ^{2} k}
\end{aligned}
$$

The coefficients $\beta_{9}, \beta_{10}$ and $\beta_{11}$ need not be calculated because they only contribute to the coefficient of $A$ in the amplitude equation (3.23), and this coefficient follows readily from the (exact) dispersion relalion (2.10). The corresponding equalions and boundary conditions for $\psi^{-}$and $f^{-}$will nol be needed below; they are oblained from (3.14)-(3.18) using the transformation

$$
\psi^{+} \rightarrow \psi^{-}, \quad f^{+} \rightarrow f^{-}, \quad \psi^{\mathrm{m}} \rightarrow-\psi^{\mathrm{m}}, \quad \Omega^{\mathrm{m}} \rightarrow-\Omega^{\mathrm{m}}, \quad A \leftrightarrow B, \quad x \rightarrow-x,
$$

a consequence of the symmetry of Fqs. (2.1)-(2.5) under the reflection $x \rightarrow-x$.

In view of the condilion (3.5) the terms on the right-hand side of Fqs. (3.14)-(3.18) are to be considered as ithomogencous, while those on the left constitule a sel of homogencous equalions solved by $\left(\psi^{+}, f^{+}\right)=\left(\psi_{0}(y), 1\right)$. The solvability condilion for this system yields the evolulion equalion for $A$ (the amplimale equation) in the form

$$
(\mathrm{i} k)^{-1} \Psi_{0}^{\prime}(0) H_{1}-(\mathrm{i} \omega)^{-1} \Psi_{0}(0) H_{2}+\Psi_{0}^{\prime}(-1) H_{3}=\int_{-1}^{0} \Psi_{0}(y) H_{0}(y) \mathrm{d} y
$$

Here $I_{0}, I_{1}, I_{2}$ and $I_{3}$ denote the right hand sides of Lqs. (3.14)-(3.17), respectively. Using (3.8) this relation takes the explicit form

$$
\begin{aligned}
A_{t}-v_{g} A_{x}= & \left.\mathrm{i} \alpha A_{x x}-\mathrm{L}(1+\mathrm{i}) \alpha_{1} C_{g}^{1 / 2}+\alpha_{2} C_{g}\right] A+\mathrm{i}\left(\alpha_{3}|A|^{2}-\alpha_{4}|B|^{2}\right) A+\mathrm{i} \alpha_{5} \mu \bar{B} \\
& +\mathrm{i} \alpha_{6} \int_{-1}^{0} g(y)\left\langle\psi_{y}^{m}\right\rangle^{x} \mathrm{~d} y A+\mathrm{i} \alpha_{7}\left\langle f^{m}\right\rangle^{x} A+\text { HO' }^{\prime} .
\end{aligned}
$$

To use this procedure to compute the coefficients of $A_{x x}$ and $C_{g} A$, we would have to consider the expansions

$$
\psi^{+}=A_{x} \psi_{1}^{+}+C_{g}^{1 / 2} A \psi_{2}^{+}+\cdots, \quad f^{+}=A_{x} f_{1}^{+}+C_{g}^{1 / 2} A f_{2}^{+}+\cdots,
$$

and explicitly calculate $\left(\psi_{1}^{+}, f_{1}^{+}\right)$and $\left(\psi_{2}^{+}, f_{2}^{+}\right)$from the equations that result from substituting these expansions into (3.8), (3.14)-(3.18) and selting the coefficients of $A_{x}$ and $C_{y}^{1 / 2} A$ lo zero; note thal condition (3.8) is necessary to ensure utiqueness in these singular problems. In praclice, it is simpler to deduce the coeflicients $\alpha_{1}$ and $\alpha_{2}$, the group velocily vg and the dispersion $\alpha$ direclly from the dispersion relation (2.10) using (2.11) and

$$
\nu_{\mathrm{g}}=\omega^{\prime}(k), \quad \alpha=-\frac{1}{2} \omega^{\prime \prime}(k)
$$

The remainitig coeflicients in (3.23), $\alpha_{3}, \ldots, \alpha_{7}$ must, however, be calculaled from the solvability condition (3.22) atid are found to be 


$$
\begin{aligned}
& \alpha_{3}=\frac{\omega k^{2}\left[(1-S)\left(9-\sigma^{2}\right)\left(1-\sigma^{2}\right)+S k^{2}\left(7-\sigma^{2}\right)\left(3-\sigma^{2}\right)\right]}{4 \sigma^{2}\left[(1-S) \sigma^{2}-S k^{2}\left(3-\sigma^{2}\right)\right]}+\frac{\left[8(1-S)+5 S k^{2}\right\rceil \omega k^{2}}{4\left(1-S+S k^{2}\right)}, \\
& \alpha_{4}=\frac{\omega k^{2}}{2}\left[\frac{\left(1-S+S k^{2}\right)\left(1+\sigma^{2}\right)^{2}}{\left(1-S+4 S k^{2}\right) \sigma^{2}}+\frac{\left.4(1-S)+7 S k^{2}\right]}{1-S+S k^{2}}\right], \quad \alpha_{5}=\omega k \sigma, \quad \alpha_{6}=\frac{k \sigma}{2 \omega}, \\
& \alpha_{7}=\frac{\omega k\left(1-\sigma^{2}\right)}{2 \sigma} .
\end{aligned}
$$

These expressions agree up to notation changes with their counterparts in strictly inviscid theories (see, e.g., [12] and references therein), and in particular confirm the results for the cubic coefficients obtained in Refs. $[4,27]$. Like $\gamma_{2}$ and $\gamma_{3}$, the coefficient $\alpha_{3}$ diverges at the (excluded) resonant wavenumbers satisfying $\omega(2 k)=2 \omega(k)$. The corresponding amplitude equation for the complex amplitude $B$ is obtained from (3.23) using the reflection symmetry (3.21).

The resulting equations take the form (1.1) and (1.2) if we select a (large) integer $N$ such that

$$
-\pi<2 \pi N-k I \leq \pi,
$$

and replace

$$
A \rightarrow A \mathrm{e}^{\mathrm{i}(2 \pi N / L-k) x}, \quad B \rightarrow B \mathrm{e}^{-\mathrm{i}(2 \pi N / T \cdot-k) x},
$$

and redefine $v_{\mathrm{g}}$ to be the group velocity at the wavenumber $2 \pi N / L$. This change of variables shifts the wavenumber $k$ to the nearest wavenumber commensurate with the imposed periodicity condition and leads to periodic boundary conditions on the (new) variables $A$ and $B$ as in (1.3); the resulting expressions for the damping rate $\delta$ and the effective detuning $d$ present in Lis. (1.1) and (1.2) are given by (2.12) and

$$
d=\alpha_{1} c_{g}^{1 / 2}-\left(\frac{2 \pi N}{L}-k\right) v_{g} .
$$

Both quantities are small. The change of variables has, however, no effect on the mean flow equations (1.4)-(1.9), except to replace the wavenumber $k$ that appears explicitly in the boundary condition (1.7b) by $k=2 \pi N / L$, i.e., the solution in the bulk is now of the form

$$
\begin{aligned}
& f=\mathrm{e}^{\mathrm{i} w / t /}\left[\left(A \mathrm{e}^{\mathrm{i} 2 \pi N x / L}+B \mathrm{e}^{-\mathrm{i} 2 \pi N x / L}\right)+\mathrm{HOI}\right]+\text { c.c. }+f^{\mathrm{m}}+\mathrm{NR}^{\prime} \mathrm{I}, \\
& \psi=\mathrm{e}^{\mathrm{i} \omega t}\left[\Psi_{0}\left(\Lambda \mathrm{e}^{\mathrm{i} 2 \pi N x / L}-B \mathrm{e}^{-\mathrm{i} 2 \pi N x / L}\right)+\mathrm{IIOT}\right]+\text { c.c. }+\psi^{\mathrm{m}}+\mathrm{NRT}, \\
& \Omega=\Omega^{\mathrm{ml}}+\text { IIOT. }
\end{aligned}
$$

Some remarks about the GCAMF equalions derived above are now in order.

(a) The conservalive nalure of the terms describing the coupling to the mean flow implies that at leading order the meat flow does nol take cnergy from the system, a result that is consistenl. with the small slecpness of the associated surface displacement and its small velocily compared wilh the speed $|\nabla \psi|$ of the surface waves. This latter properly follows from the fact. that the mean flow is driven by quadratic terms in the complex amplitudes (see remarks (b) and (c)).

(b) The forcing lerms on the right-hand sides of the boundary condilions (1.5a) and (1.6) are presenl. when the aspect ralio is large, and are responsible for driving the striclly inviseid mean flow $[11,12]$. These terms vanish if the waveltain is uniform, or if the surface waves are of standing wave lype, namely if $|A|=|B|$, and $k \gg 1$ (so that $\left.\beta_{3} \ll 1\right)$. Note that part of the striclly intviscid mean flow cant be included explicilly in the expansion (3.2) because it is slaved to the waves. However, we choose not to do so here because there is always a part of this now which 
solves a homogeneous problem (see Eq. (4.12)) and is not slaved. The shear nature of the remaining forcing terms, in Eqs. (1.5b) and (1.7c), leads us to retain the viscous term in (1.4) even when $C_{g}$ is quite small. In fact, when $C_{g}$ is very small, the mean flow itself generates additional boundary layers near the top and bottom of the container, and these must be thicker than the original boundary layers for the validity of the analysis. This puts an additional restriction on the validity of the GCAMF equations, namely

$$
\left|\frac{\beta_{2} k\left(|A|^{2}-|B|^{2}\right)}{C_{g}}\right|^{1 / 3}+\left|\frac{\beta_{4} k\left(|A|^{2}+|B|^{2}\right)}{C_{g}}\right|^{1 / 2} \ll\left(\frac{C_{g}}{\omega}\right)^{1 / 2} .
$$

In this expression the first term is an estimate of the inverse of the boundary layer thickness associated with the tangential stress boundary condition at the surface while the second term is the corresponding quantity due to the horizontal velocity boundary condition at $y=-1$.

(c) There is a third, less effective but inviscid, volumetric forcing mechanism associated with the second term in the vorticity equation (1.4), which looks like a horizontal force $\left(|A|^{2}-|B|^{2}\right) g(y) \Omega^{\mathrm{m}}$ and is sometimes called the vortex force. The term plays an important role in the generation of Langmuir circulation [28]. Although in the absence of mean flow this term vanishes, it can change the stability properties of such a flow and enhance or limit the effect of the remaining forcing terms. However, this is not the case in the limit considered here.

(d) The GCAMF equations (1.1)-(1.9) are invariant under reflection

$$
\psi^{\mathrm{m}} \rightarrow-\psi^{\mathrm{m}}, \quad \Omega^{\mathrm{m}} \rightarrow-\Omega^{\mathrm{m}}, \quad A \leftrightarrow B, \quad x \rightarrow-x .
$$

The simplest reflection-symmetric solutions, i.e., solutions of the form $A(x, \cdot)=B(-x, \cdot)$, are the spatially uniform standing waves given by $A=B=R_{0} \mathrm{e}^{\mathrm{i} / \prime}$, where $\theta$ is a constant and the amplitude $R_{0}$ is given by $\delta^{2}+\left[d+\left(\alpha_{3}-\right.\right.$ $\left.\alpha_{4}\right) R_{0}^{2} I^{2}=\alpha_{5}^{2} \mu^{2}$, with an associated reflection-symmetric streaming flow that is periodic in $x$ with period $\pi / k$ (see Eq. (1.7c)). Since this mean flow does not couple to the amplitudes $A, B$ (i.e., the mean flow terms are absent from Eqs. (1.1) and (1.2)), the presence of this flow does not affect the standing waves. These much studied waves bifurcate from the flat state at $\mu=\mu_{\mathrm{c}}=\left(\delta^{2}+d^{2}\right)^{1 / 2} /\left|\alpha_{5}\right|$, and do so supercritically if $d<0$ and subcritically if $d>0$, see Fig. 3. Note that $\mu$ can be of order $\mu_{\mathrm{c}}$ without violating the conditions for the validity of the GCAMF equations, and that these equations describe correctly both cases $d<0$ and $d>0$. In the former case, the waves are stable near threshold, but may lose stability at finite amplitude through the action of the mean flow as the forcing amplitude increases. Like the secondary saddle-node bifurcation which stabilizes the spatially uniform standing waves when $d>0$ (see Fig. 3), this bifurcation is well within in the regime of validity of the GCAMF equations. Thus the mean flow is involved only in possible secondary instabilities of the primary standing wave branch.

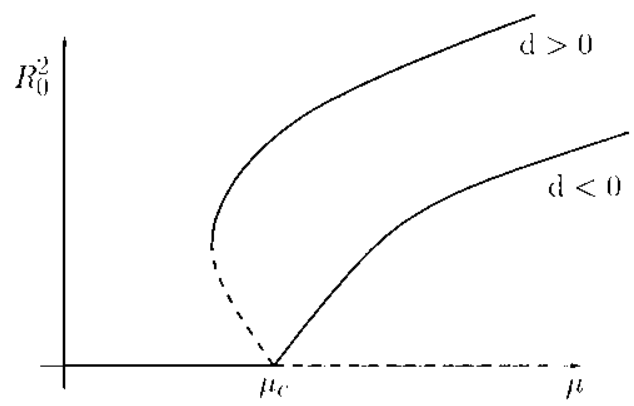

Fig. 3. The primary bifurcation from the flat state to the spatially uniform standing wave solutions. The GCAMF equations describe correctly all states with $\left|\ell-\mu_{c}\right| \sim \mu_{c}$, including the secondary saddle-node bifurcation present when $d>0$ and the solutions beyond it. 
(e) 'The special case $d=0$ (zero detuning) and $\mu=\mu_{\mathrm{c}}$ defines a codimension-two point for the analysis since both $L$ (or equivalently $\omega$ ) and $\mu$ must be chosen appropriately. In this case the direction of branching is determined by higher order terms neglected in the analysis, such as the real parts of the coefficients of the cubic terms, and this is so for sufficiently small but nonzero values of $d$ as well. In other words, the limit $d \rightarrow 0$ (although well-defined within the GCAMF equations) may not describe correctly the corresponding behavior of the underlying fluid equations appropriately close to threshold, i.e., for $\left|\mu-\mu_{c}\right| \ll \mu_{c}$. IIowever, even in this case the GCAMF equations will correctly capture any secondary instabilities involving the mean flow, provided these occur at $\mu \sim \mu_{c}$. A similar remark applies to other codimension-two points as well.

(f) The GCAMF equations form a good starting point for any weakly nonlinear theory under the assumptions $(2.8),(2.16),(2.18),(3.4)$ and (3.32), provided that second and third order internal resonances are avoided, and higher order terms consistently omitted. In fact, the condition (3.4) can be replaced by

$$
\left|A_{x}\right| \ll k|A|, \quad\left|A_{i}\right| \ll \omega|A|, \quad\left|B_{x}\right| \ll k|B|, \quad\left|B_{i}\right| \ll \omega|B|,
$$

where $A, B$ are themselves small. From the derivation of these equations it is clear that they apply whenever the parameters $C_{g}, N^{-1}$ (or $L^{-1}$ ) and $\mu$ are small, but are otherwise unrelated to one another. Any relation between them, such as those assumed in Sections 4.1 and 4.2, will therefore lead to further simplifications.

(g) If an additional packet of nearly inviscid modes is present initially, with a basic frequency $\bar{\omega} \neq \omega$ and sufficiently distinct wavenumber $\vec{k}$ (see assumption (2.16)), the associated complex amplitudes interact with the original according to the equations

$$
\begin{aligned}
\tilde{A}_{t}-\tilde{\nu}_{\mathrm{g}} \tilde{A}_{x}= & \mathrm{i} \tilde{\alpha} \tilde{A}_{x x}-(\tilde{\delta}+\mathrm{i} \tilde{d}) \tilde{A}+\mathrm{i}\left(\tilde{\alpha}_{3}|\tilde{A}|^{2}-\tilde{\alpha}_{4}|\tilde{B}|^{2}+\tilde{\alpha}_{s}|A|^{2}-\tilde{\alpha}_{9}|B|^{2}\right) \tilde{A}+\mathrm{i} \tilde{\alpha}_{6} \int_{-1}^{0} \tilde{g}(y)\left\langle\psi_{y}^{\mathrm{m}}\right\rangle^{x} \mathrm{~d} y \tilde{A} \\
& +\mathrm{i} \tilde{\alpha}_{7}\left(f^{\mathrm{m}}\right\rangle^{x} \tilde{A}+\mathrm{IIOT}, \\
\tilde{B}_{t}+\tilde{\nu}_{\mathrm{g}} \tilde{B}_{x}= & \mathrm{i} \alpha \tilde{B}_{x x}-(\tilde{\delta}+\mathrm{i} \tilde{d}) \tilde{B}+\mathrm{i}\left(\tilde{\alpha}_{3}|\tilde{B}|^{2}-\tilde{\alpha}_{4}|\tilde{A}|^{2}+\tilde{\alpha}_{8}|B|^{2}-\tilde{\alpha}_{9}|A|^{2}\right) \tilde{B}-\mathrm{i} \tilde{\alpha}_{6} \int_{-1}^{0} \tilde{g}(y)\left(\psi_{y}^{\mathrm{m}}\right\rangle^{x} \mathrm{~d} y \tilde{B} \\
& +\mathrm{i} \tilde{\alpha}_{7}\left\langle\int^{\mathrm{m}}\right\rangle^{x} \tilde{B}+\mathrm{HOT}, \\
\tilde{A}(x+L, t) \equiv & \tilde{A}(x, t), \quad \tilde{B}(x+L, t) \equiv \tilde{B}(x, t),
\end{aligned}
$$

i.e., such modes evolve under the influence of the ambient wavetrain, but are not directly excited by the parametric forcing. It is clear from the structure of these equations that this interaction cannot maintain the additional packet against viscous dissipation, and hence that both $\tilde{A}$ and $\tilde{B}$ decay exponentially on the timescale $t \sim \tilde{\delta}^{-1}$.

\section{Coupled amplitude-mean flow equations for moderately large aspect ratios}

The regime $1 \ll L \ll v_{\mathrm{g}} /(\delta+|d|+\mu)$ provides perhaps the cleanest simplification of the GC.AMF equations derived above. The resulling equations apply to systems of moderalely large aspecl ratios, and include in a particular regime the model equalions studied al length by Martel el al. [17]. To derive such simplified equations we consider the distinguished limil

$$
\frac{\delta L^{2}}{\alpha}=\Delta \sim 1, \quad \frac{d L^{2}}{\alpha}=D \sim 1, \quad \frac{\mu L^{2}}{\alpha} \equiv M \sim 1
$$

with $1 \leqslant k \lesssim\left|\ln C_{g}\right|$. Fq. (2.12) then implies that $C_{g}\left|\ln C_{g}\right|^{2} \lesssim \delta \lesssim C_{g}^{1 / 2}$. In order to avoid unnecessarily involved expressions, we shall henceforth |real $\left|\ln C_{g}\right|$ as an $O(1)$ quantily, thereby allowing some of the coefficients in the 
expansions (4.8), (4.9), (4.48) and (4.49) to be Iogarithmically small or large. 'The simplified equations are derived using a multiple scale method using $x$ and $t$ as fast variables and

$$
\zeta=\frac{x}{L}, \quad \tau=\frac{t}{L}, \quad T=\frac{t}{L^{2}},
$$

as slow variables. In terms of these variables the local horizontal average ()$^{x}$ defited in (3.19) becomes an average over the fast variable $x$. Note that assumption (4.1) imposes an implicil relation between $L$ and $C_{g}$. In the following, we distinguish two sub-limils, depending on whether gravily is significant $(1-S \sim 1)$ or negligible $(1-S \ll 1)$. The resulting equations are valid in the whole range $1 \ll L \ll \nu_{\mathrm{g}} /(\delta+|d|+\mu)$, and more specifically ror $1 \ll L \ll C_{g}^{-1 / 2}$ if $k \sim 1$.

\subsection{Gravity or gravity-capillary waves}

When $1-S \sim 1$ the nearly inviscid and viscous mean flows can be clearly distinguished from one another as discussed in Section 2, and the viscous mean flow can be identified by taking appropriate averages of the whole mean flow over an intermediate timescale $\tau$, i.e., the mean flow variables $\psi^{\mathrm{m}}, \Omega^{\mathrm{m}}$ and $f^{\mathrm{m}}$ take the form

$$
\begin{aligned}
& \psi^{\mathrm{m}}(x, y, \zeta, \tau, T)=\psi^{v}(x, y, \zeta, T)+\psi^{i}(x, y, \zeta, \tau, T), \\
& \Omega^{\mathrm{m}}(x, y, \zeta, \tau, T)=\Omega^{v}(x, y, \zeta, T)+\Omega^{i}(x, y, \zeta, \tau, T), \\
& f^{\mathrm{m}}(x, \zeta, \tau, T)=f^{v}(x, \zeta, T)+f^{i}(x, \zeta, \tau, T)
\end{aligned}
$$

with

$$
\left|\int_{0}^{\tau} \psi_{x}^{i} \mathrm{~d} \tau\right|+\left|\int_{0}^{\tau} \psi_{\zeta}^{i} \mathrm{~d} \tau\right|+\left|\int_{0}^{\tau} \psi_{y}^{i} \mathrm{~d} \tau\right|+\left|\int_{0}^{\tau} \Omega^{i} \mathrm{~d} \tau\right|+\left|\int_{0}^{\tau} f^{i} \mathrm{~d} \tau\right|
$$

bounded as $\tau \rightarrow \infty$. Thus the nearly inviscid mean flow is purely oscillatory (i.e., it has a zero mean, see (4.6)) on the timescale $\tau$. Since its frequency is of the order of $L^{-1}$ (see (4.2)), which is large compared with $C_{g}$, the inertial term for this flow is large in comparison with the viscous terms (see Lq. (1.4)), except in two secondary boundary layers, of thickness of the order of $\left(C_{g} L\right)^{1 / 2}(\ll 1)$, attached to the bottom plate and the free surface. Note that, as required for the consistency of the analysis, these boundary layers are much thicker than the prinary boundary layers associated with the surface waves (see lig. 2), which provide the boundary conditions (1.5)-(1.7) for the mean flow. Moreover, the width of these secondary boundary layers remains small as $\tau \rightarrow \infty$ and (to leading order) the vorticity of this nearly inviscid mean flow remains confined to these boundary layers. 'This is because, according to condition (4.6), the nearly inviscid mean flow is purely oscillatory on the timescale $\tau$. Consequently, condition (4.6) is essential for the validity of the analysis that follows, and the mathematical definition of the nearly inviscid mean flow through Liqs. (4.3)-(4.6) is the only consistent one; without this condition vorticity would diffuse outside the boundary layers and affect the structure of the whole 'nearly inviscid' solution even at leading order. In fact, vorticity does diffuse (and is convected) from the boundary layers, but this vorticity transport is included in the viscous mean flow. The vorticity associated with the nearly inviscid mean flow is readily seen to be of, at most, the order of

$$
\left.|| A\right|^{2}-|B|^{2} \mid, \quad\left(|A|^{2}+|B|^{2}\right)\left(C_{g} L\right)^{-1 / 2}
$$

in the upper and lower secondary boundary layers, respectively; the jump it the associaled streamfunction $\psi^{i}$ across each boundary layer is $O\left(C_{g} L\right)$ limes smaller. This jump only affects higher order terms; as a consequence the secondary boundary layers can be completely ignored and no additional contributions to the boundary conditions 
on the nearly inviscid flow need be included in (1.5) and (1.7). Outside these boundary layers, the complex amplitudes and the flow variables associated with the nearly inviscid mean flow are expanded as

$$
\begin{aligned}
& (A, B)=L^{-1}\left(X_{0}, Y_{0}\right)+L^{-2}\left(X_{1}, Y_{1}\right)+\cdots, \quad\left(\psi^{i}, f^{i}\right)=L^{-2}\left(\phi_{0}^{i}, F_{0}^{i}\right)+L^{-3}\left(\phi_{1}^{i}, F_{1}^{i}\right)+\cdots, \\
& \Omega^{i}=L^{-3} W_{0}^{i}+\cdots, \quad\left(\psi^{v}, \Omega^{v}\right)=L^{-2}\left(\phi_{0}^{v}, W_{0}^{v}\right)+\cdots, \quad f^{v}=L^{-3} F_{0}^{v}+\cdots .
\end{aligned}
$$

Substitution of (4.1)-(4.6), (4.8) and (4.9) into (1.1)-(1.9) leads to the following:

(i) Irom (1.4)-(1.7) at leading order

$$
\phi_{0 x x}^{i}+\phi_{0 y y}^{i}=0 \quad \text { in }-1<y<0, \quad \phi_{0}^{i}=0 \quad \text { at } y=-1, \quad \phi_{0 x}^{i}=0 \quad \text { at } y=0,
$$

together with $F_{0 x}^{i}=0$. Thus

$$
\phi_{0}^{i}=(y+1) \Phi_{0}^{i}(\zeta, \tau, T), \quad F_{0}^{i}=F_{0}^{i}(\zeta, \tau, T)
$$

At second order, the boundary conditions $(1.5 \mathrm{a})$ and $(1.6)$ yield

$$
\begin{aligned}
& \phi_{1 x}^{i}(x, 0, \zeta, \tau, T)=F_{0 \tau}^{i}-\Phi_{0 \zeta}^{i}+\beta_{1}\left(\left|Y_{0}\right|^{2}-\left|X_{0}\right|^{2}\right)_{\zeta}, \\
& (1-S) F_{1 x}^{i}-S F_{1 x x x}^{i}=\Phi_{0 \tau}^{i}-(1-S) F_{0 \zeta}^{i}-\beta_{3}\left(\left|X_{0}\right|^{2}+\left|Y_{0}\right|^{2}\right)_{\zeta}
\end{aligned}
$$

at $y=0$. Since the right-hand sides of these two equalions are independent. of the last variable $x$ and both $\phi_{1}^{i}$ and $F_{1}^{i}$ must be bounded in $x$, il follows that:

$$
\Phi_{0 \zeta}^{i}-F_{0 \tau}^{i}=\beta_{1}\left(\left|Y_{0}\right|^{2}-\left|X_{0}\right|^{2}\right)_{\zeta}, \quad \Phi_{0 \tau}^{i}-v_{\mathrm{p}}^{2} F_{0 \zeta}^{i}=\beta_{3}\left(\left|X_{0}\right|^{2}+\left|Y_{0}\right|^{2}\right)_{\zeta},
$$

where

$$
\nu_{\mathrm{p}}=(1-S)^{1 / 2}
$$

is the phase velocity of long wavelength surface gravity waves. Lq. (4.12) must be integrated with the following additional conditions, which result from (1.8), (1.9) and (4.6),

$$
\begin{aligned}
& \Phi_{0}^{i}(\zeta+1, \tau, T) \equiv \Phi_{0}^{i}(\zeta, \tau, T), \quad F_{0}^{i}(\zeta+1, \tau, T) \equiv F_{0}^{i}(\zeta, \tau, T) \\
& \int_{0}^{1} F_{0}^{i} \mathrm{~d} \zeta=0, \quad\left|\int_{0}^{\tau} \Phi_{0 \zeta}^{i} \mathrm{~d} \tau\right|+\left|\int_{0}^{\tau} F_{0}^{i} \mathrm{~d} \tau\right|=\text { bounded as } \tau \rightarrow \infty
\end{aligned}
$$

(ii) The leading order contributions to Eqs. (1.1) and (1.2) yield $X_{0 \tau}-v_{\mathrm{g}} X_{0 \zeta}=Y_{0 \tau}+v_{\mathrm{g}} Y_{0 \zeta}=0$. Thus

$$
X_{0}=X_{0}(\xi, T), \quad Y_{0}=Y_{0}(\eta, T),
$$

where $\xi$ and $\eta$ are the characteristic variables

$$
\xi=\zeta+v_{\mathrm{g}} \tau, \quad \eta=\zeta-v_{\mathrm{g}} \tau
$$

Moreover, according to (1.3)

$$
X_{0}(\xi+1, T) \equiv X_{0}(\xi, T), \quad Y_{0}(\eta+1, T) \equiv Y_{0}(\eta, T) .
$$

Substitulion of these expressions intlo (4.12) followed by inlegration of the resulting equations yields

$$
\left.\Phi_{0}^{i}=\left.\frac{\beta_{1} v_{\mathrm{p}}^{2}+\beta_{3} v_{\mathrm{g}}}{v_{\mathrm{g}}^{2}-v_{\mathrm{p}}^{2}}|| X_{0}\right|^{2}-\left|Y_{0}\right|^{2}-\left\langle\left|X_{0}\right|^{2}-\left|Y_{0}\right|^{2}\right\rangle^{\zeta}\right]+v_{\mathrm{p}}\left[F^{+}\left(\zeta+v_{\mathrm{p}} \tau, T\right)-F^{-}\left(\zeta-v_{\mathrm{p}} \tau, T\right)\right],
$$




$$
\left.F_{0}^{i}=\frac{\beta_{1} v_{\mathrm{o}}+\beta_{3}}{v_{\underline{g}}^{2}-v_{\mathrm{p}}^{2}}\left\lceil\left|X_{0}\right|^{2}+\left|Y_{0}\right|^{2}-\left\langle\left|X_{0}\right|^{2}+\left|Y_{0}\right|^{2}\right\rangle^{\zeta}\right]+F^{+}\left(\zeta+v_{\mathrm{p}} \tau, T\right)+F^{-}\left(\zeta-v_{\mathrm{p}} \tau, T\right)\right],
$$

where ()$^{\zeta}$ denoles the mean value over the slow spatial variable $\zeta$, i.c.,

$$
\langle G\rangle^{\zeta}=\int_{0}^{1} G \mathrm{~d} \zeta
$$

and the functions $F^{ \pm}$are such that

$$
F^{ \pm}\left(\zeta+1 \pm v_{p} \tau, T\right) \equiv F^{ \pm}\left(\zeta \pm v_{p} \tau, T\right), \quad\left\langle F^{ \pm}\right\rangle^{\zeta}=0
$$

The particular solution of (4.19) and (4.20) yields the usual inviscid mean flow included in nearly inviscid theories (see [12] and references therein); the averaged terms are a consequence of the conditions (4.15), i.e., of volume conservation (cf. [12]) and the requirement that the nearly inviscid mean flow has a zero mean on the timescale $\tau$; the latter condition is never imposed in strictly inviscid theories but is essential in the limit we are considering, as explained above. To avoid the breakdown of the solution (4.19) and (4.20) at $v_{\mathrm{p}}=v_{\mathrm{g}}$, we assume in addition that

$$
\left|v_{\mathrm{p}}-v_{\mathrm{g}}\right| \sim 1 .
$$

The functions $F^{ \pm}$remain undetermined at this stage. In fact, they are not needed below because the evolution of both the viscous mean flow and the complex amplitudes is decoupled from these functions. Ilowever, at next order one finds that $F^{ \pm}$remain constant on the timescale $T$, but decay exponentially due to viscous effects (resulting from viscous dissipation in the secondary boundary layer attached to the bottom plate) on the timescale $t \sim\left(L_{/} / C_{g}\right)^{1 / 2}$.

(iii) The evolution equations for $X_{0}$ and $Y_{0}$ on the timescale $T$ are readily obtained from Eqs. (1.1)-(1.3), invoking $(4.1)-(4.6),(4.19),(4.20)$ and (4.22) and eliminating secular terms (i.e., requiring $\left|X_{1}\right|$ and $\left|Y_{1}\right|$ to be bounded on the timescale $\tau$ ):

$$
\begin{aligned}
& X_{O T}=\mathrm{i} \alpha X_{0 \xi \xi}-(\Delta+\mathrm{i} D) X_{0}+\mathrm{i}\left[\left(\alpha_{3}+\alpha_{s}\right)\left|X_{0}\right|^{2}-\alpha_{s}\left\langle\left|X_{0}\right|^{2}\right\rangle^{\xi}-\alpha_{4}\left\langle\left|Y_{0}\right|^{2}\right\rangle^{\eta}\right] X_{0}+\mathrm{i} \alpha_{5} M\left(\bar{Y}_{0}\right\rangle^{\eta} \\
& +\mathrm{i} \alpha_{6} \int_{-1}^{0} g(y)\left\langle\left(\phi_{0 y}^{v}\right)^{x}\right\rangle^{\zeta} \mathrm{d} y X_{0} \\
& Y_{O T}=\mathrm{i} \alpha Y_{O \eta \eta}-(\Delta+\mathrm{i} D) Y_{0}+\mathrm{i}\left[\left(\alpha_{3}+\alpha_{8}\right)\left|Y_{0}\right|^{2}-\alpha_{8}\left(\left|Y_{0}\right|^{2}\right\rangle^{\eta}-\alpha_{4}\left\langle\left|X_{0}\right|^{2}\right\rangle^{\xi} \mid Y_{0}+\mathrm{i} \alpha_{5} M\left(\bar{X}_{0}\right)^{\xi}\right. \\
& -\mathrm{i} \alpha_{6} \int_{-1}^{0} g(y)\left\langle\left\langle\phi_{0 y}^{v}\right\rangle^{x}\right\rangle^{\zeta} \mathrm{d} y Y_{0} \\
& X_{0}(\xi+1, T) \equiv X_{0}(\xi, T), \quad Y_{0}(\eta+1, T) \equiv Y_{0}(\eta, T) .
\end{aligned}
$$

Here $\xi$ and $\eta$ are the comoving variables defined in (4.17), and $\langle\cdot\rangle^{x},\langle\cdot\rangle^{\zeta},\langle\cdot\rangle^{\xi}$ and $\langle\cdot\rangle^{\eta}$ denote mean values over the variables $x, \zeta, \xi$ and $\eta$, respectively. Note that $\zeta$ averages over functions of $X_{0}$ are equivalent to $\xi$ averages, while those over functions of $Y_{0}$ are equivalent to $\eta$ averages. The real coefficient $\alpha_{8}$ is given by

$$
\alpha_{8}=\frac{\alpha_{6}(2 \omega / \sigma)\left(\beta_{1} v_{\mathrm{p}}^{2}+\beta_{3} v_{\mathrm{g}}\right)+\alpha_{7}\left(\beta_{1} v_{\mathrm{g}}+\beta_{3}\right)}{v_{\mathrm{g}}^{2}-v_{\mathrm{p}}^{2}}
$$

Eqs. (4.24) and (4.25) are independent of $F^{ \pm}$because of the second condition in (4.22).

Eqs. (4.24) and (4.25) depend on the horizontal velocity of the viscous mean flow, $-\phi_{0 y}^{\prime \prime}$. The equations and boundary conditions governing the evolution of this flow are derived by substituting Eqs. (4.3)-(4.6), (4.8)-(4.11), (4.19) and (4.20) into (1.4)-(1.7), obtaining

$$
\phi_{0 x x}^{v}+\phi_{0 y y}^{v}=W_{0}^{v},
$$




$$
\begin{aligned}
& \left.W_{0 T}^{v}-\mid \phi_{0 y}^{v}+\left\langle\left|X_{0}\right|^{2}-\left|Y_{0}\right|^{2}\right\rangle^{\zeta} g(y)\right] W_{0 x}^{v}+\phi_{0 x}^{v} W_{0 y}^{v}=R e^{-1}\left(W_{0 x x}^{v}+W_{0 y y}^{v}\right) \quad \text { in }-1<y<0, \\
& \phi_{0 x}^{v}=0, \quad \phi_{0 y y}^{v}=\beta_{2}\left\langle\left|X_{0}\right|^{2}-\left|Y_{0}\right|^{2}\right\rangle^{\tau} \quad \text { at } y=0, \\
& \left\langle\left\langle W_{0 y}^{v}\right\rangle^{x}\right\rangle^{\zeta}=\phi_{0}^{v}=0, \quad \phi_{0 y}^{v}=-\beta_{4}\left[\mathrm{i}\left\langle X_{0} \bar{Y}_{0}\right\rangle^{\tau} \mathrm{e}^{\mathrm{i} 4 \pi N x / L}+\text { c.c. }+\left\langle\left|Y_{0}\right|^{2}-\left|X_{0}\right|^{2}\right\rangle^{T}\right] \quad \text { at } y=-1, \\
& \phi_{0}^{v}(x+L, \zeta+1, y, T) \equiv \phi_{0}^{v}(x, \zeta, y, T),
\end{aligned}
$$

where $X_{0} \equiv X_{0}\left(\zeta+v_{\mathrm{g}} \tau, T\right), Y_{0} \equiv Y_{0}\left(\zeta-v_{\mathrm{g}} \tau, T\right)$ are given by (4.24) and (4.25) and ()$^{\tau}$ denotes averages over the limescalc $\tau$. The effecive Reynolds number associaled with this viscous mean flow is

$$
R e=\frac{1}{C_{g} L^{2}} .
$$

Some remarks aboul these equalions and boundary conditions are now ifl order.

1. The viscous mean flow is associated with only a small free-surface deflection, $f^{v} \sim L^{-3}$ (see (4.9)), which plays no role in the evolution of this flow, as expected of a flow involving the excitation of viscous modes (see Section 2).

2. According to the scaling (4.1) and the definitions (2.11), (2.12) and (4.33), the effective Reynolds number $R e$ is large, and ranges from logarithmically large values if $k \sim\left|\ln C_{g}\right|$ to $\mathrm{O}\left(C_{g}^{-1 / 2}\right)$ if $k \sim 1$. IIowever, even in the latter limit we must retain the viscous terms in (4.29) in order to account for the second boundary conditions in (4.30) and (4.31). Of course, if $R e \gg 1$ vorticity diffusion is likely to be confined to thin layers, but the structure and location of all these layers cannot be anticipated in an obvious way (see below) and in this case we must rely on numerical computations for realistically large values of $R e$.

3. The viscous mean flow is driven by the short gravity-capillary waves through the averaged terms in the boundary conditions (4.30) and (4.31). The quantity $\left\langle\left|X_{0}\right|^{2}-\left|Y_{0}\right|^{2}\right\rangle^{\tau}=\left\langle\left|X_{0}\right|^{2}\right\rangle^{\xi}-\left\langle\left|Y_{0}\right|^{2}\right\rangle^{\prime \prime}$ (=0, see below) depends only on $T$, but $\left\langle X_{0} \bar{Y}_{0}\right\rangle^{T}$ (which will play a major role below) depends on both $\zeta$ and $T$ (unless either $X_{0}$ or $Y_{0}$ is spatially uniform). Thus, because of the boundary condition (4.31), $\phi_{0}^{v}$ and $W_{0}^{v}$ depend on both the fast and slow horizontal spatial variables $x$ and $\zeta$. Unfortunately, the dependence of $\phi_{0}^{0}$ and $W_{0}^{v}$ on $x$ cannot be obtained in closed form (except, of course, in the uninteresting limit $R e \rightarrow 0$ ), and we must rely, once again, on numerical computations for realistically large values of $L$.

4. Observe that the boundary conditions $(4.30 \mathrm{~b})$ and $(4.31 \mathrm{c})$ contain inhomogeneous forcing terms that are averages over the intermediate timescale $\tau$. Like the oscillatory terms $F^{ \pm}$in Eqs. (4.19) and (4.20) the omitted terms oscillate on this limescale and hence generate secondary boundary layers. The contribulions from these boundary layers are all subdomitant and have no efrect at the order considered.

5. The dominanl. forcing of the viscous mean flow comes from the lower boundary. This forcing vanishes exponenlially when $k \gg 1$ leaving only a narrow range of wavenumbers wilhin which such a mean flow is forced while $\delta=O\left(C_{g}\right)$, see Fig. 1. Thus in most. cases in which viscous mean flow is present. one may assume that $\delta=O\left(C_{g}^{1 / 2}\right)$. Note, however, that in fully three-dimensional siluations (such as that in [291) int which lateral walls are included a viscous mean now will be presenl even when $k \gg 1$ because the forcing of the mean flow in the oscillatory boundary layers altached to the lateral walls remains.

The form of the parametric forcing terms in (4.24) and (4.25) allows a further simplification of the system (4.24), (4.25), (4.28)-(4.32). With the change of variables

$$
X_{0}=\tilde{X}_{0} \mathrm{e}^{-2 \pi \mathrm{i} N O / L}, \quad Y_{0}=\bar{Y}_{0} \mathrm{e}^{2 \pi \mathrm{i} N O / L},
$$


where $\theta \equiv \theta(T)$ obeys

$$
o^{\prime}(T)=-(2 \pi N)^{-1} L \alpha_{0} \int_{-1}^{0} g(y)\left\langle\left(\phi_{0 y}^{v}\right)^{x}\right\rangle^{\zeta} \mathrm{d} y
$$

the mean flow decouples, and Fqs. (4.24)-(4.26) become

$$
\begin{aligned}
& \tilde{X}_{0 T}=\mathrm{i} \alpha \tilde{X}_{0 \xi \xi}-(\Delta+\mathrm{i} D) \tilde{X}_{0}+\mathrm{i}\left[\left(\alpha_{3}+\alpha_{8}\right)\left|\tilde{X}_{0}\right|^{2}-\alpha_{8}\left\langle\left|\tilde{X}_{0}\right|^{2}\right\rangle^{\xi}-\alpha_{4}\left\{\left|\tilde{Y}_{0}\right|^{2}\right\rangle^{\eta} \mid \tilde{X}_{0}+\mathrm{i} \alpha_{5} M\left\langle\tilde{\tilde{Y}}_{0}\right\}^{\eta},\right. \\
& \tilde{Y}_{O T}=\mathrm{i} \alpha \tilde{Y}_{0 \eta \eta}-(\Delta+\mathrm{i} D) \tilde{Y}_{0}+\mathrm{i}\left[\left(\alpha_{3}+\alpha_{8}\right)\left|\tilde{Y}_{0}\right|^{2}-\alpha_{8}\left\langle\left|\tilde{Y}_{0}\right|^{2}\right\rangle^{\eta}-\alpha_{4}\left\{\left|\tilde{X}_{0}\right|^{2}\right\rangle^{\xi}\right] \tilde{Y}_{0}+\mathrm{i} \alpha_{5} M\left\langle\overline{\tilde{X}}_{0}\right\}^{\xi}, \\
& \tilde{X}_{0}(\xi+1, T) \equiv \tilde{X}_{0}(\xi, T), \quad \tilde{Y}_{0}(\eta+1, T) \equiv \tilde{Y}_{0}(\eta, T) .
\end{aligned}
$$

Lxcept for differences in notation these equations are identical to the equations already extensively investigated by Martel et al. [17]. In constructing their nonlocal amplitude equations Martel et al. deliberately ignored the possible presence of viscous mean flow in order to write down a tractable system of equations. Consequently, they considered their equations to be a phenomenological description of the liaraday system rather than a quantitatively precise one. The present paper shows that the equations originally written down in Ref. [17] do in fact provide a quantitative description of this system, and establishes the conditions under which they do so. In addition, the systematic derivation of these equations indicates that the omitted viscous mean flow does in fact play a role in that it affects the spatial phase of the pattern, and the manner in which it does so. In view of the exact relation

$$
\frac{\mathrm{d}\left\langle\left(\left.\tilde{X}_{0}\right|^{2}-\left|\tilde{Y}_{0}\right|^{2}\right\rangle^{\zeta}\right.}{\mathrm{d} T}=-2 \Delta\left\langle\left|\tilde{X}_{0}\right|^{2}-\left|\tilde{Y}_{0}\right|^{2}\right\rangle^{\zeta}, \quad \Delta>0,
$$

we may assume that

$$
\left\langle\left|\tilde{X}_{0}\right|^{2}-\left|\bar{Y}_{0}\right|^{2}\right\rangle^{\zeta} \equiv\left\langle\left|\tilde{X}_{O}\right|^{2}\right\rangle^{\xi}-\left\langle\left|\tilde{Y}_{0}\right|^{2}\right\rangle^{\eta}=0,
$$

and rewrite the viscous mean flow equations (4.28)-(4.32) in the form

$$
\begin{aligned}
& \phi_{0 x x}^{v}+\phi_{0 y y}^{v}=W_{0}^{v}, \\
& W_{0 T}^{v}-\phi_{0 y}^{v} W_{0 x}^{v}+\phi_{0 x}^{v} W_{0 y}^{v}=R e^{-1}\left(W_{0 x x}^{v}+W_{0 y y}^{v}\right) \quad \text { in }-1<y<0, \\
& \phi_{0 x}^{v}=\phi_{0 y y}^{v}=0 \quad \text { at } y=0, \\
& \left\langle\left(W_{0 y}^{v}\right)^{x}\right\rangle^{\zeta}=\phi_{0}^{v}=0, \quad \phi_{0 y}^{v}=2 \beta_{4} R_{0}(\zeta, T) \sin \left[\frac{4 \pi N\left(x-\theta-\theta_{0}\right)}{L}\right] \text { at } y=-1, \\
& \phi_{0}^{v}(x+L, \zeta+1, y, T) \equiv \phi_{0}^{v}(x, \zeta, y, T),
\end{aligned}
$$

where the functions $R_{0}=R_{0}(\zeta, T)$ and $\theta_{0}=\theta_{0}(\zeta, T)$ are defined by

$$
R_{0} \mathrm{e}^{-4 \pi \mathrm{i} N \theta_{0} / I}=\left\langle\tilde{X}_{0} \overline{\tilde{Y}}_{0}\right\rangle^{r} \equiv \sum_{-\infty}^{+\infty} x_{n}(T) \bar{y}_{-n}(T) \mathrm{e}^{4 \pi \mathrm{i} n \zeta}
$$

and $\left\{x_{n}\right\},\left\{y_{n}\right\}$ are the Fourier coefficients in the expansions

$$
\tilde{X}_{0}(\xi, T)=\sum_{-\infty}^{+\infty} x_{n}(T) \mathrm{e}^{2 \pi \mathrm{i} n \xi}, \quad \tilde{Y}_{0}(\eta, T)=\sum_{-\infty}^{+\infty} y_{n}(T) \mathrm{e}^{2 \pi \mathrm{i} n \eta}
$$

Fqs. (4.35), (4.40)-(4.44) describe the resul ling coupled cvolution of the spatial phase $\theta$ of the pallerti and of the viscous mean flow, and conslitute a separate dynamical system forced by the amplitude dynamies studied by Marlel 
et al. [17] via the functions $R_{0}$ and $\theta_{0}$ appearing in the boundary condition (4.43). Note that the viscous mean flow is forced by the bottom boundary layer only, and that this forcing vanishes exponentially when $k \gg 1$ (see remarks (3) and (5) above).

It is worth remarking that the condition (4.39) prevents the existence of spatially uniform progressive waves (i.e., solutions of the type $|A|=$ constant, $|B|=$ constant, $|A| \neq|B|$ ) as solutions of the nonlocal equations (4.36) and (4.37). I Iowever, a number of other solution types is possible. These split naturally into solutions lying in the invariant subspace $|A|=|B|$ and those with $|A| \neq|B|$. With periodic boundary conditions the former can be either symmetric with respect to a spatial reflection $x \rightarrow-x$ or nonsymmetric. As discussed in more detail by Martel et al. [17] solutions of the former type may be uniform and steady, nonuniform and steady, time-periodic and chaotic. The same is also true for the nonsymmetric solutions, but in this case the spatial asymmetry is responsible for the presence of a net drift of the solution. This is a consequence of the periodic boundary conditions (4.38), and introduces an additional, typically small frequency into the solution. Drifts of this type are called type I in order to distinguish them from type II drifts that are due to an asymmetry between the amplitudes of left- and right-traveling wavetrains. Type II drifts are present even if both $|A|$ and $|B|$ are reflection-symmetric about some point $x$ (not necessarily the same), provided only that $|\Lambda| \neq|B|$, modulo translation. Moreover, when both of these two types of asymmetry are present multiply periodic drifts will result, as discussed and illustrated by Martel et al. As a result the variety of possible solutions to even the simplest set of equations, the decoupled amplitude equations, is quite substantial, and each such solution is accompanied in addition by a viscous mean flow. This mean flow responds to

type I drifts in the amplitudes $\bar{X}_{0}, \tilde{Y}_{0}$ through the amplitude $R_{0}$, and to type II drifts through the dependence of the forcing on the phase $O_{0}$. The explicit computation of the relevant coefficients performed in this paper can be used to identify physically relevant regimes in the classification of Ref. [17].

\subsection{Capillary waves in the microgravity limit}

As $1-S \rightarrow 0$, the phase velocity of the long (gravity) waves $v_{\mathrm{p}}$ vanishes (see Lq. (4.13)) and a part of the nearly inviscid mean flow defined above resonates with the viscous mean flow. In fact, because of the decomposition (4.3)-(4.7) this resonant interaction is captured completely in the viscous mean flow, which will now involve a significant free surface deformation.

We suppose that

$$
(1-S) L^{2}=A \sim 1,
$$

and consider the expansions

$$
\begin{aligned}
& (A, B)=L^{-1}\left(X_{0}, Y_{0}\right)+L^{-2}\left(X_{1}, Y_{1}\right)+\cdots, \quad\left(\psi^{i}, f^{i}\right)=L^{-2}\left(\phi_{0}^{i}, F_{0}^{i}\right)+L^{-3}\left(\phi_{1}^{i}, F_{1}^{i}\right)+\cdots \\
& \Omega^{i}=L^{-3} W_{0}^{i}+\cdots, \quad\left(\psi^{v}, \Omega^{v}\right)=L^{-2}\left(\phi_{0}^{v}, W_{0}^{v}\right)+\cdots, \quad f^{v}=L^{-1} F_{0}^{v}+\cdots
\end{aligned}
$$

The resulting analysis proceeds as in Section 4.1. The main differences are that Lqs. (4.12), (4.19) and (4.20) must be replaced, respectively, by

$$
\begin{aligned}
& \Phi_{0 \zeta}^{i}-F_{0 \tau}^{i}=\beta_{1}\left(\left|Y_{0}\right|^{2}-\left|X_{0}\right|^{2}\right\rangle_{\zeta}, \quad \Phi_{0 \tau}^{i}=\beta_{3}\left(\left|X_{0}\right|^{2}+\left|Y_{0}\right|^{2}\right\rangle_{\zeta}, \\
& \left.\Phi_{0}^{i}=\left(\frac{\beta_{3}}{v_{0}}\right) \Gamma\left|X_{0}\right|^{2}-\left|Y_{0}\right|^{2}-\left\langle\left|X_{0}\right|^{2}-\left|Y_{0}\right|^{2}\right\rangle^{\zeta}\right], \\
& F_{0}^{i}=\left(\frac{\beta_{1} v_{g}+\beta_{3}}{v_{g}^{2}}\right)\left[\left|X_{0}\right|^{2}+\left|Y_{0}\right|^{2}-\left\langle\left|X_{0}\right|^{2}+\left|Y_{0}\right|^{2}\right\rangle^{\zeta}\right] .
\end{aligned}
$$


Because of the conditions (4.15) the solution of the homogeneous part of (4.50) vanishes identically. Thus $X_{0}$ and $Y_{0}$ are still given by (4.24)-(4.26), but $S$ must now be replaced by 1 everywhere in the expressions for the coefficients $\alpha_{3}, \ldots, \alpha_{6}$ and $\alpha_{s}$, and the parameters $\Delta, D$ and $M$. Eqs. (4.28) and (4.29) and the conditions (4.31) and (4.32) still apply, but the boundary conditions (4.30) must be replaced by

$$
\phi_{0 x}^{v}=F_{0 T}^{v}, \quad \phi_{0 y y}^{v}=\beta_{2}\left\langle\left|X_{0}\right|^{2}-\left|Y_{0}\right|^{2}\right\rangle^{\tau} \quad \text { at } y=0 .
$$

Moreover, Prom (1.6)

$$
\phi_{0 y T}^{v}=\Lambda F_{O \zeta}^{v}-F_{0 \zeta \zeta \zeta}^{v} \quad \text { at } y=0 .
$$

If we redefine the complex amplitudes as in (4.34) the amplitude equations (4.36)-(4.38) still uncouple from the viscous mean flow, and for large times (on the timescale $T$ ) the result (4.39) implies that the phase shift $\theta$ and the viscous mean flow evolve according to

$$
\begin{aligned}
& 0^{\prime}(T)=-(2 \pi N)^{-1} L \alpha_{6} \int_{-1}^{0} g(y)\left\langle\left(\phi_{0 y}^{v}\right)^{x}\right\rangle^{\zeta} \mathrm{d} y, \\
& \phi_{0 x x}^{v}+\phi_{0 y y}^{v}=W_{0}^{v}, \\
& W_{0 T}^{v}-\phi_{0 y}^{v} W_{0 x}^{v}+\phi_{0 x}^{v} W_{0 y}^{v}=R e^{-1}\left(W_{0 x x}^{v}+W_{0 y y}^{v}\right) \quad \text { in }-1<y<0, \\
& \phi_{0 x}^{v}=F_{0 T}^{v}, \quad \phi_{0 y y}^{v}=0, \quad \phi_{0 y T}^{v}=\Lambda F_{0 \zeta}^{v}-F_{\zeta \zeta \zeta}^{v} \quad \text { at } y=0, \\
& \left\langle\left(W_{0 y}^{v}\right)^{x}\right\rangle^{\zeta}=\phi_{0}^{v}=0, \quad \phi_{0 y}^{v}=2 \beta_{4} R_{0}(\zeta, T) \sin \left[\frac{4 \pi N\left(x-\theta-\theta_{0}\right)}{L}\right] \quad \text { at } y=-1, \\
& \phi_{0}^{v}(x+L, \zeta+1, y, T) \equiv \phi_{0}^{v}(x, \zeta, y, T)
\end{aligned}
$$

with the functions $R_{0}=R_{0}(\zeta, T)$ and $O_{0}=O_{0}(\zeta, T)$ still given by (4.45) and (4.46) in terms of the solutions of the decoupled system (4.36)-(4.38). Thus the structure of the problem in the microgravity limit and in the presence of gravity is fundamentally the same, and the study of the decoupled system (4.36)-(4.38) by Martel et al. [17] applies to both.

\section{Concluding remarks}

In this paper, we have given a systemalic derivation of the basic equalions governing the inleraction belween paramelrically exciled surlace gravily-capillary waves in nearly inviscid fluids and a meatl hlow. We have argued that in such fluids, depending on the aspect ratio of the container, the hydrodynamic (or bulk) modes decay more slowly than the surface waves and that such modes cannol therefore be omilled from a consistenl. weakly nonlitiear description of these systems. Since the excitation of these modes manifests itself as a (viscous) mean flow a description in terms of equalions of the lype summarized in (1.1)-(1.9) is inevilable; we delcrmined here explicilly the condilions under which this is the case. In general, traveling waves are associaled wilh the presence of an inviscid mean flow as well [12], and, consequenlly, the (total) mean flow it these equations intcludes contributions from both sources. Under the condilions of Section 4 , the viscous mean flow is drivent by a langential velocily boundary condition imposed on the largely inviscid flow in the bulk. This boundary condition deseribes the net. efrect. on the bulk of the presence of atl oscillatory viscous boundary layer altached to the boltom of the container, as first discussed by Schlichting [20]. In general, we found that the lower boundary is more effeclive at driving the viscous meat now than a similar boundary layer at the free surface which provides a stress boundary condition on the mean 
flow in the bulk [21]. In contrast, the purely inviscid flow that may be present is a consequence of the mechanism by which the waves are excited [30].

A careful examination of the analysis that led us to Eqs. (1.1)-(1.9) shows that these in fact apply under the conditions

$$
k\left(\left|\psi_{x}\right|+\left|\psi_{y}\right|\right) \ll \omega, \quad|f|+\left|f_{x}\right| \ll 1, \quad L^{-1} \ll k,
$$

or equivalently

$$
k(|A|+|B|)+\left|f_{x}^{\mathrm{m}}\right| \ll 1, \quad k\left|\psi_{x}^{\mathrm{m}}\right| \ll \omega,
$$

obtained from (2.17), and the condition

$$
L \ll \frac{v_{g}}{\delta+|d|+\left|\alpha_{5}\right| \mu}
$$

that relaxes somewhat the requirement (2.19). Here $v_{\mathrm{g}}$ is the (nondimensional) group velocily of the surface waves, defined in (3.24), $\alpha_{5}$ is given in (3.25) and we assumed that the smallest spatial scale is $k^{-1}$. The condition (5.1) can be stated succinclly as requiring that the nonlinearily be weak and the aspect ratio of the system be large compared to the nondimensional wavelength of the surface waves; the condition (5.3) requires that the terms accounting for incria and propagation at the group velocily in the amplitude equations (1.1) and (1.2) be much larger than the remaining terms. In addition, the requirements

$$
(1-S) k^{2}+S k^{4} \gg C_{g}^{2}, \quad k^{3 / 2}\left(1-S+S k^{2}\right)^{-1 / 2} \ll C_{g}^{-1},
$$

or equivalently

$$
C_{g} \ll \omega, \quad C_{g}^{1 / 2}()^{3 / 2} \ll 1-S+\frac{S \omega}{C_{g}},
$$

are imposed implicitly both on the carrier wavenumber $k$ as well as on all wavenumbers associated with the (viscous) mean flow. These conditions guarantee that the thickness of the associated boundary layers will be small compared to the depth (if $k \ll 1$ ) or compared to the wavelength (if $k \gg 1$ ), see Fig. 2. Since the lowest wavenumber of the mean flow is $k=2 \pi / L$, condition (5.4) implies, in particular, that

$$
(1-S) L^{-2}+(2 \pi)^{2} S L^{-4} \gg C_{g}^{2} .
$$

Additional assumplions, such as the requirement that all second and third resontances are avoided (Wilton ripples) and that in Section $4 v_{\mathrm{p}} \neq v_{\mathrm{g}}$, appear in the course of the antalysis.

The resulting GCAMF equations were derived with one further but essential assumplion, namely that the spalial Fourier transforms of both the basic wavetrains and of the associaled mean flow remain peaked around a sel of discrele wavenumbers (two in the case of the carrier wavenumbers, and infittite in the case of the mean flow) for all lime. This assumption concerns the small scale struclure of the solution and il excludes the generation of small scales that may arise if the aspect ratio is 1.00 large. In fact, il is nol necessary to assume that these wavenumbers are commensurate; it is only necessary that some scale separation is present so that the averages introduced are well defined. In a numerical solulion starling with given initial condilions this assumption may either fail, indicating that the aspect ratio is 1.00 large or be found to hold for timescales of interest. The equalions derived here deseribe the latter siluation. We have also seen that under cerlain specific conditions it is possible to distinguish unambiguously the two lypes of mean flow (viscous and inviscid), and described in Section 4 a parlicularly useful instance in which this can be done. The equalions derived there by meats of at additional multiple scale analysis led to a surprisingly simple description of the resulting system, consisting of a pair of decoupled, albeil nonlocal equations of the lype 
already studied at length in [17], together with a set of equations governing the interaction of the spatial phase of the wave amplitudes and the viscous mean flow. Since the Reynolds number of this flow can be (indeed must be) substantial these equations must be treated numerically as already done in other circumstances [31,32]. Such solutions will be reported elsewhere. To the extent the presence of lateral walls may be ignored the results may provide a cluantitative description of the plethora of experimental results on the Faraday system with nearly inviscid fluids $[3,14,29,33]$.

It is useful to consider an explicit experimental realization of the theory described here. We focus on an annular container with a $110 \mathrm{~mm}$ diameter filled with extremely clean (see [34] and references therein) water to an $8 \mathrm{~mm}$ depth (as in [29]), but with a forcing frequency of $10.6 \mathrm{IIz}$. Using $T=72 \mathrm{dyn} / \mathrm{cm}$, we calculate the gravity-capillary time (see Section 2) to be $0.027 \mathrm{~s}$ and hence that $\omega=0.87$. The remaining dimensionless parameters of the theory then take the values $L=43.2, S=0.1, C_{g}=4.2 \times 10^{-4}, k=1, \delta=3.8 \times 10^{-3}, d=-0.0102$ and $v_{g}=0.767$.

Under these conditions the requirements $(2.17)-(2.19)$ for the validity of the theory are fulfilled, and twodimensional waves of small steepness are described by the GCAMF equations derived above. An experiment of the above type, designed to minimize three-dimensional effects, could therefore test the predictions of these equations.

\section{Acknowledgements}

This work was supported by the Nalional Acronaulies and Space $\Lambda$ dministration under Grant NAC3-2152 and by the Spanish Direceión General de Enseñanza Superior under Grant PB97-0556 (CM and JMV).

\section{Appendix A. Boundary conditions on the bulk flow}

\section{A.1. Stokes boundary layer near the bottom plate}

lor convenience, we take the small parameter $\varepsilon$ as the order of magnitude of the complex amplitudes, namely, we assume that

$$
|A| \sim|B| \sim \varepsilon, \quad\left|\psi^{\mathrm{m}}\right| \sim \varepsilon^{2}
$$

in (3.2). We introduce the stretched coordinate

$$
\tilde{y}=\frac{y+1}{C_{g}^{1 / 2}}
$$

and seck a solution in the form

$$
\left(C_{g}^{-1 / 2} \psi, C_{g}^{1 / 2} \Omega\right)=\varepsilon\left(\tilde{\psi}_{1}, \tilde{\Omega}_{1}\right) \mathrm{c}^{\mathrm{i} \omega l}+\text { c.c. }+\varepsilon^{2}\left\lceil\left(\tilde{\psi}_{2}, \tilde{\Omega}_{2}\right)+\mathrm{OT}\right\rceil+\cdots,
$$

where for $j=1$ and $2, \tilde{\psi}_{j}$ and $\tilde{\Omega}_{j}$ depend weakly on time and $O$ T stands for oscillatory terms on the time scale $t \sim 1$. Substitution of $(\Lambda .2)$ and $(\Lambda .3)$ into (2.1) and (2.4) yiclds

$$
\begin{aligned}
& \bar{\psi}_{1 y y}=\bar{\Omega}_{1}, \quad \Omega_{1 \hat{y} y}=\mathrm{i} \omega \Omega_{1} \quad \text { in } 0<\bar{y}<\infty, \quad \tilde{\psi}_{1}=\tilde{\psi}_{1 y}=0 \quad \text { at } \tilde{y}=0, \\
& \tilde{\psi}_{2 \hat{y} y}=\tilde{\Omega}_{2}, \quad \tilde{\Omega}_{2 \hat{y} \hat{y}}=-\overline{\tilde{\psi}}_{1 \hat{y}} \tilde{\Omega}_{1 x}+\tilde{\psi}_{1 x} \overline{\tilde{\Omega}}_{1 \hat{y}}+\text { c.c. } \quad \text { in } 0<\bar{y}<\infty, \\
& \bar{\psi}_{2}=\tilde{\psi}_{2 \check{y}}=0 \text { al } \tilde{y}=0 \text {, }
\end{aligned}
$$


where the overbar stands for the complex conjugate. In addition, we have

$$
\tilde{\Omega}_{1}=\tilde{\Omega}_{2}=0 \quad \text { as } \tilde{y} \rightarrow \infty
$$

as required by matching with the solution in the bulk, which is completed below. Integration of (A.4) yields

$$
\tilde{\psi}_{1}=K_{1}(x) \frac{\sqrt{i \omega} \tilde{y}+\exp (-\sqrt{i \omega} \tilde{y})-1}{\sqrt{\mathrm{i} \omega}}
$$

where ( $\Lambda .6)$ has been taken into accounl:; $K_{1}(x)$ is determined by malching condilions between (3.2) and $(\Lambda .3)$ at order $\varepsilon$ ( $\sec$ also $(\Lambda .1)$ and $(\Lambda .2))$ and is given by

$$
\varepsilon K_{1}=\Psi_{0}^{\prime}(-1)\left(A \mathrm{e}^{\mathrm{i} k x}-B \mathrm{e}^{-\mathrm{i} k x}\right)
$$

Substitulion into the second equation $(\Lambda .5)$ now yields

$$
\tilde{\Omega}_{2 \bar{y} \bar{y}}=\sqrt{\mathrm{i} \omega} \bar{K}_{1} K_{|x|}\left[-\mathrm{c}^{-\sqrt{\mathrm{i} \omega} \bar{y}}+(-1+\sqrt{\mathrm{i} \omega} \bar{y}) \mathrm{c}^{-\sqrt{-\mathrm{i} \omega} \bar{y}}+2 \mathrm{c}^{-\sqrt{2 \omega} \bar{y}} 1+\mathrm{c.c} .\right.
$$

and we need only inlegrate this equation twice, using $(\Lambda .6)$, and inlegrate $(\Lambda .5 \mathrm{a})$ once, using $(\Lambda .5 \mathrm{c})$, 10 oblaitl

$$
\bar{\psi}_{2 \overline{\mathrm{y}}}=\bar{K}_{1} K_{1 x}\left[\frac{3-3 \mathrm{i}}{2 \omega}+\frac{\mathrm{e}^{-\sqrt{\mathrm{i} \omega} \bar{y}}}{\mathrm{i} \omega}+\left(\frac{3 \mathrm{i}-1}{\omega}+\frac{\tilde{y}}{\sqrt{-\mathrm{i} \omega}}\right) \mathrm{e}^{-\sqrt{-\mathrm{i} \omega} \bar{y}}-\frac{1+\mathrm{i}}{2 \omega} \mathrm{c}^{-\sqrt{2 \omega} \tilde{y}}\right]+\text { c.c. }
$$

With this expression for $\tilde{\psi}_{2 \tilde{y}}$, we can apply matching conditions between (3.2) and (A.3) at order $\varepsilon^{2}$ (see also (A.1), (A.2) and (A.8)) to obtain

$$
\begin{aligned}
& \psi_{x}^{\mathrm{m}}(x,-1, t)=\sigma\left(\varepsilon^{2}\right), \\
& \psi_{y}^{\mathrm{m}}(x,-1, t)=\varepsilon^{2}\left[\frac{3(1-\mathrm{i}) \bar{K}_{1} K_{1 . x}}{2 \omega}+\text { c.c. }\right]=-3 \omega k\left(1-\sigma^{2}\right) \sigma^{-2}\left(\mathrm{i} A \bar{B} \mathrm{c}^{\mathrm{zi} k x}+\text { c.c. }+|B|^{2}-|A|^{2}\right),
\end{aligned}
$$

where we have used the relation $\Psi_{0}^{\prime}(-1)^{2}=\omega^{2}\left(1-\sigma^{2}\right) \sigma^{-2}$ obtained from (2.13). The boundary condition (1.7b) now follows with $\beta_{4}$ as given in (3.20).

\section{A.2. Oscillatory boundary layer near the free surface}

l'or convenience, we assume again that (A.1) holds, introduce a stretched coordinate attached to the interface

$$
\tilde{y}=\frac{y-f(x, l)}{C_{g}^{1 / 2}},
$$

and seek a solution in the form

$$
\begin{aligned}
& \psi=\varepsilon\left(\tilde{\psi}_{1}+C_{g}^{1 / 2} \tilde{\psi}_{2}+C_{g} \tilde{\psi}_{3}\right) \mathrm{e}^{\mathrm{i} \omega / t}+\text { c.c. }+\varepsilon^{2}\left(\tilde{\psi}_{4}+C_{g}^{1 / 2} \tilde{\psi}_{5}+C_{g} \tilde{\psi}_{6}+\mathrm{OT}\right)+\cdots, \\
& \Omega=\varepsilon\left(\tilde{\Omega}_{1}+\cdots\right) \mathrm{c}^{\mathrm{i} \omega l}+\text { c.c. }+\varepsilon^{2}\left(\tilde{\Omega}_{2}+\cdots+\mathrm{OT}\right)+\cdots,
\end{aligned}
$$

i.c., we anlicipale that. $\Omega \sim \varepsilon$. We also rewrite (3.1) in the form

$$
f=\varepsilon\left(f_{1}+C_{g}^{1 / 2} f_{2}\right) \mathrm{e}^{\mathrm{i} \omega t}+\text { c.c. }+\cdots .
$$

Substitulion of $(\Lambda .12)-(\Lambda .14)$ inlo (2.1) and (2.2) now yiclds the following system of equations and boundary 
conditions:

(A) Ior the oscillatory part of (A.13)

$$
\begin{aligned}
& \tilde{\psi}_{1 \tilde{y} y}=\tilde{\psi}_{2 \tilde{y} y}=\tilde{\psi}_{3 \tilde{y} y}+\tilde{\psi}_{1 x x}-\tilde{\Omega}_{1}=\tilde{\Omega}_{1 \tilde{y} \tilde{y}}-\mathrm{i} \omega \tilde{\Omega}_{1}=0 \quad \text { in }-\infty<\tilde{y}<0, \\
& \bar{\psi}_{1 . x}-\mathrm{i} \omega f_{1}=\bar{\psi}_{2 x}-\mathrm{i} \omega f_{2}=\bar{\psi}_{3 y y}-\tilde{\psi}_{1 x x}=0 \quad \text { at } \tilde{y}=0, \\
& \bar{\psi}_{1 y}=\tilde{\Omega}_{1}=0 \quad \text { as } \tilde{y} \rightarrow-\infty .
\end{aligned}
$$

Thus

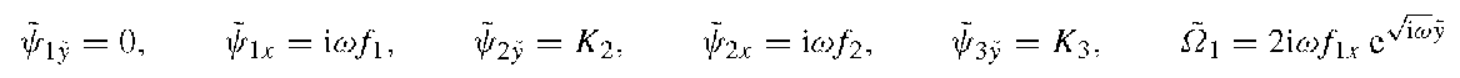

wilh $K_{2}$ and $K_{3}$ independent of $\tilde{y}$.

(B) For the slowly varying part of ( $\Lambda .13)$

$$
\begin{aligned}
& \tilde{\psi}_{4 y \hat{y}}=\tilde{\psi}_{5 y \hat{y}}=\tilde{\psi}_{6 \hat{y} \hat{y}}+\tilde{\psi}_{4 x x}-\tilde{\Omega}_{2}-\left(2 \bar{f}_{1 x} K_{2 x}+\bar{f}_{1 x x} K_{2}+\text { c.c. }\right)=0 \text { in }-\infty<\tilde{y}<0, \\
& \tilde{\Omega}_{2 \hat{y} \hat{y}}+\left(\bar{K}_{2} \tilde{\Omega}_{1 . x}-\bar{y} \bar{K}_{2 x} \tilde{\Omega}_{1 \hat{y}}+c . c .\right)=0 \quad \text { in }-\infty<\tilde{y}<0, \\
& \bar{\psi}_{4 x}=\bar{\psi}_{6 y \hat{y}}-\bar{\psi}_{4 x x}+\left(-2 \bar{f}_{1 x} K_{2 x}+\bar{f}_{1 x x} K_{2}+\text { c.c. }\right)=0 \quad \text { al } \tilde{y}=0, \\
& \bar{\psi}_{4 \bar{y}}=\bar{\Omega}_{2 \tilde{y}}=0 \quad \text { as } \tilde{y} \rightarrow-\infty
\end{aligned}
$$

where we have taken into account (A.18). Thus

$$
\begin{aligned}
& \bar{\psi}_{4}=0, \quad \bar{\psi}_{5 \hat{y}}=K_{4}, \\
& \tilde{\psi}_{6 \tilde{y} \dot{y}}=2\left\lfloor\bar{K}_{2 x} f_{1 x}(\sqrt{i \omega} \tilde{y}-2)-f_{1 x x} \bar{K}_{2}\right\rfloor \mathrm{e}^{\sqrt{ } \mathrm{i} \omega \tilde{y}}+f_{1 x x} \bar{K}_{2}+6 f_{1 x} \bar{K}_{2 x}+\text { c.c. },
\end{aligned}
$$

where $K_{4}$ is again independent of $\tilde{y}$. lior matching with the solution in the bulk we need the $\tilde{y} \rightarrow-\infty$ limit of horizontal velocity and stress:

$$
\begin{aligned}
\psi_{y}(x, f, t) & =\varepsilon \tilde{\psi}_{2 y} \mathrm{e}^{\mathrm{i} \omega t}+\text { c.c. }+\varepsilon^{2}\left(\tilde{\psi}_{5 y}+\mathrm{OT}\right)+\cdots=\varepsilon K_{2} \mathrm{e}^{\mathrm{i} \omega t}+\text { c.c. }+\varepsilon^{2}\left(K_{4}+\mathrm{OT}\right)+\cdots, \\
\psi_{y y}(x, f, t) & =\varepsilon \tilde{\psi}_{3 y} \mathrm{e}^{\mathrm{i} \omega t}+\text { c.c. }+\varepsilon^{2}\left(\tilde{\psi}_{6 y}+O \mathrm{OI}\right)+\cdots \\
& =\varepsilon K_{3} \mathrm{e}^{\mathrm{i} \omega t t}+\text { c.c. }+\varepsilon^{2}\left(6 \bar{f}_{1 x} K_{2 x}+\bar{f}_{1 x x} K_{2}+\text { c.c. }+O \mathrm{O}\right)+\cdots
\end{aligned}
$$

On the other hand, for the solution in the bulk $\psi_{y}(x, f(x, t), t)=\psi_{y}(x, 0, t)+\cdots$ and $\psi_{y y}(x, f(x, t), t)=$ $\psi_{y y}(x, 0, t)+\psi_{y y y}(x, 0, t) f(x, t)+\cdots$ or, according to (3.1) and (3.2)

$$
\begin{aligned}
\psi_{y}(x, f, t)= & \Psi_{0}^{\prime}(0) \mathrm{e}^{\mathrm{i} \omega t}\left(A \mathrm{e}^{\mathrm{i} k x}-B \mathrm{e}^{-\mathrm{i} k x}\right)+\text { c.c. }+\cdots \\
\psi_{y y}(x, f, t)= & \Psi_{0}^{\prime \prime}(0) \mathrm{c}^{\mathrm{i} \omega t}\left(A \mathrm{e}^{\mathrm{i} k x}-B \mathrm{e}^{-\mathrm{i} k x}\right)+\text { c.c. } \\
& +\left[\Psi_{0}^{\prime \prime \prime}(0)\left(A \mathrm{e}^{\mathrm{i} k x}-B \mathrm{e}^{-\mathrm{i} k x}\right)\left(\bar{A} \mathrm{e}^{-\mathrm{i} k x}+\bar{B} \mathrm{e}^{\mathrm{i} k x}\right)+\text { c.c. }+\psi_{y y}^{\prime m}(x, 0, t)+O \mathrm{I}^{\prime}\right]+\cdots
\end{aligned}
$$

Identification of (3.1) with (A.14) and (A.23) with (A.25) yields

$$
\varepsilon f_{1}=A \mathrm{e}^{\mathrm{i} k x}+B \mathrm{e}^{-\mathrm{i} k x}, \quad \varepsilon K_{2}=\Psi_{0}^{\prime}(0)\left(A \mathrm{e}^{\mathrm{i} k x}-B \mathrm{e}^{-\mathrm{i} k x}\right) .
$$


lïnally, matching expressions (A.24) with (A.26) gives

$$
\begin{aligned}
& \psi_{y}^{\mathrm{m}}(x, 0, t)=\left[5 k^{2} \Psi_{0}^{\prime}(0)-\Psi_{0}^{\prime \prime \prime}(0)\right]\left(A \mathrm{e}^{\mathrm{i} k x}-B \mathrm{e}^{-\mathrm{i} k x}\right)\left(\bar{A} \mathrm{e}^{\mathrm{i} k x}+\bar{B} \mathrm{e}^{-\mathrm{i} k x}\right)+c \cdot c .=\frac{8 \omega k^{2}\left(|A|^{2}-|B|^{2}\right)}{\sigma}, \\
& \psi_{x}^{\mathrm{m}}(x, 0, t)=o\left(\varepsilon^{2}\right),
\end{aligned}
$$

and hence the coefficient $\beta_{2}$ in $\mathrm{Eq}$. (3.20).

\section{References}

[1] I. Miles, 1). Henderson, Parametrically forced surface waves, Annu. Rev. Fluid Mech. 22 (1990) $143-165$.

[2] S. Fauye, Parametric inslabilities, in: G. Martínez Mekler, T.II. Seligman (Eds.). Dynamics of Nonlinear and Disordered Systems, World Scientific, Singapore, 1995, pp. 67-115.

[3] A. Kudrolli, J.P. Gollub, Patterns and spatio-temporal chaos in parametrically forced surface waves: a systematic survey at large aspect ratio, Physica D 97 (1997) 133-154.

[4] P.l.. Hansen, P. Alstrom, Perturbation theory of parametrically driven capillary waves at low viscosity, J. Hluid . Mech. 351 (1997) 301-344.

[5] C.. Martel, H. Knobloch, D)amping of nearly inviscid water waves, Phys. Rev. H. 56 (1997) $5544-5548$.

[6] (.. Martel, J.A. Nicolás, J.M. Vega, Surface-wave damping in a brimful cylindrical container, .1. Huid Mech. 360)(1998) 213-228, sec also Corrigendum 373 (1998) 379 .

[7] D.M. Ilenderson, I.W. Miles, Surface-wave damping in a circular cylinder with a fixed contact line, J. Гluid Mech. 275 (1994) $285-299$.

[81 D.M. Henderson, J.W. Miles, $A$ note on interior vs. boundary layer damping of surface waves in a circular cylinder, J. Fluid Mech. 365 (1998) 89-107.

[9] J.A. Nicolás, J.M. Vega, Weakly nonlinear oscillations of axisymmetric liquid bridges, J. Fluid Mech. 328 (1996) 95-128.

[10] M. Higuera, I.A. Nicolás, J.M. Vega, Coupled amplitude-strcaming flow equations for the cvolution of counter-rotating, nearly-inviscid surface waves in linite axisymmetric geometries, Preprint, 2000.

[11] A. Davey, K. Stewartson, On three-dimensional packets of surface waves, Proc. R. Soc. London, Ser. A 338 (1974) $101-110$.

[12] R.D. Pierce, E. Knobloch, On the modulational stability of traveling and standing water waves, Phys. Fluids 6 (1994) $1177-1190$.

[13] A. Davey, L.M. Hocking, K. Stewartson, On nonlinear evolution of three-dimensional disturbances in plane Poiseuille flow, J. Fluid Mech. 63 (1974) $529-536$.

[14] A.B. Hzerskii, M.I. Rabinovich, V.P. Reutov, I.M. Starobinets, Spatiotemporal chaos in the parametric excitation of a capillary ripple, Sov. Phys. JETP 64 (1986) 1228-1236.

[15] O.M. Phillips, The Dynamics of the Lpper Ocean, Cambridge University Press, Cambridge, 1977.

[16] A.D.D. Craik, The drift velocity of water waves, J. Fluid Mech. 116 (1982) 187-205.

[17] C. Marte1, E. Knobloch, J.M. Vega, Dynamics of counterpropagating waves in parametrically forced systems, Physica D 137 (2000) $94-123$.

[18] C.. Martel, J.M. Vega, Finite size effects near the onset of the oscillatory instability, Vonlinearity 9)(1996) $1129-1171$.

[19] (.. Martel, J.M. Vega. I ynamics of a hyperbolic system that applies at the onset of the oscillatory instability. Nonlinearity 11 (1998) $105-142$.

[20] II. Schlichting, Berechnung ebener periodischer Grenzschichtströmungen, Phys. Z. 33 (1932) 327-335.

[21] M.S. Longuet-Higgins, Mass transport in water waves, Phil. Trans. R. Soc. London, Ser. 1245 (1953) $535-581$.

[22] M.C.W. Jones, Nonlinear stability of resonant capillary-gravity waves, Wave Motion 15 (1992) 267-283.

[23] P. C'hristodoulides, F. J)ias, Resonant gravity-capillary interfacial waves, J. Hluid Mech. 265 (1994) 303-343.

[24] I..F. Mciioldrick, On Wilton ripples: a special case of resonant interactions, .l. Fluid Mech. 42 (1970) 193-200.

[25] K. Trulsen, C.C. Mei, Modulation of three resonating gravily-capillary waves by a long gravity wave. I. Fluid Mech. 290 (1995) $345-376$.

[26] K. Trulsen, C.C. Mei. Effect of weak wind and damping on Wilton's ripples, J. Гluid Mech. 335 (1997) $141-163$.

[271 J.W. Miles, On Faraday waves, J. Fluid Mech. 248 (1993) 671-683.

[281 S. Leibovich, S. Paolucci, The instability of the ocean to Langmuir circulations, J. Fluid Mech. 102 (1981) $141-167$.

[29] S. Douady. S. Hauve, O. 'Thual, Oscillatory phase modulation of parametrically forced surface waves. Furophys. $1.20 t .10$ (1989) $309-315$.

[30] F. Knobloch. R. Picrec. (On mean flows associated with travelling water waves. Fluid IJyn. Res. 22 (1998) 61-71.

[31] J.A. Nicolís, D. Rivas, J.M. Vega, The interaction of thermocapillary convection and low-frequency vibration in nearly-inviscid licpuid bridges, Z. Angew. Math. Phys. 48 (1997) 389-423.

[32] J.A. Nicolás, D. Rivas, J.M. Vega, On the steady streaming flow due to high frequency vibration in nearly-inviscid liquid bridges, J. Fluid Mech. 354 (1998) 147-174.

[33] N.B. 'Jufillaro, R. Ramshankar, I.P. (jollub, (Yrder-disorder transition in capillary ripples, Phys. Rev. $1 . e t t .62$ (1989) $422-425$.

[34] J.A. Nicolás, J.M. Vega, (In the effect of surface contamination in water wave damping, J. Hiluid . Mech. 410)(2000) 367-373. 